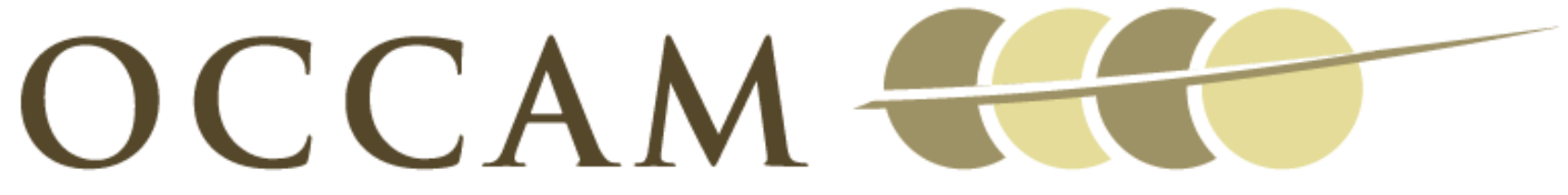

OXFORD CENTRE FOR COLLABORATIVE APPLIED MATHEMATICS

Report Number 12/21

Multiphase modelling of vascular tumour growth in two spatial dimensions

by

M.E.Hubbard and H.M.Byrne

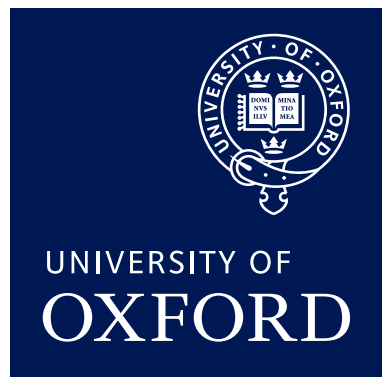

Oxford Centre for Collaborative Applied Mathematics Mathematical Institute 24 - 29 St Giles'

Oxford

OX1 3LB

England 



\title{
Multiphase modelling of vascular tumour growth in two spatial dimensions
}

\author{
M.E.Hubbard $\dagger$ \\ School of Computing, University of Leeds, Leeds, LS2 9JT, U.K. \\ †Corresponding author: m.e.hubbard@leeds.ac.uk \\ H.M.Byrne \\ Oxford Centre for Collaborative Applied Mathematics, \\ Mathematical Institute, University of Oxford, \\ 24-29 St Giles', Oxford, OX1 3LB, U.K \\ and \\ Department of Computer Science, Parks Road, Oxford, OX1 3QD, U.K.
}

February 13, 2012 


\begin{abstract}
In this paper we present a continuum mathematical model of vascular tumour growth which is based on a multiphase framework in which the tissue is decomposed into four distinct phases and the principles of conservation of mass and momentum are applied to the normal/healthy cells, tumour cells, blood vessels and extracellular material. The inclusion of a diffusible nutrient, supplied by the blood vessels, allows the vasculature to have a nonlocal influence on the other phases. Two-dimensional computational simulations are carried out on unstructured, triangular meshes to allow a natural treatment of irregular geometries, and the tumour boundary is captured as a diffuse interface on this mesh, thereby obviating the need to explicitly track the (potentially highly irregular and ill-defined) tumour boundary. A hybrid finite volume/finite element algorithm is used to discretise the continuum model: the application of a conservative, upwind, finite volume scheme to the hyperbolic mass balance equations and a finite element scheme with a stable element pair to the generalised Stokes equations derived from momentum balance, leads to a robust algorithm which does not use any form of artificial stabilisation. The use of a matrix-free Newton iteration with a finite element scheme for the nutrient reaction-diffusion equations allows full nonlinearity in the source terms of the mathematical model.

Numerical simulations reveal that this four-phase model reproduces the characteristic pattern of tumour growth in which a necrotic core forms behind an expanding rim of wellvascularised proliferating tumour cells. The simulations consistently predict linear tumour growth rates. The dependence of both the speed with which the tumour grows and the irregularity of the invading tumour front on the model parameters are investigated.
\end{abstract}




\section{Introduction}

Vascular tumour growth is a complex process, characterised by rapid cell proliferation, angiogenesis and vascular adaptation [26]. Increased rates of cell proliferation are associated with mutations that promote cell division and/or inhibit natural cell death while angiogenesis and the co-option of pre-existing blood vessels are stimulated by the production of angiogenic factors such as vascular endothelial growth factor (VEGF) that are produced by the tumour cells, often in response to hypoxia. The new and co-opted blood vessels supply the tumour with the nutrients it needs to thrive and expand [51]. Additionally, the vasculature provides tumour fragments that break away from the primary tumour with a transport network to other parts of the body where, if conditions are favourable, the tumour fragments may establish themselves as secondary tumours or metastases [25].

The spatial composition and growth rate of vascular tumours can vary markedly: some remain approximately spherical, developing a central necrotic core surrounded by a region of proliferating cells; others are irregularly shaped, with proliferating tumour cells in close proximity to densely packed blood vessels and extensive necrosis in avascular regions [21]. Developing and simulating mechanistic models that can explain and reproduce such spatiotemporal heterogeneity are major goals of mathematical and computational modellers and present serious technical challenges which this work seeks to address.

In order to place our work in context, we start by reviewing the relevant theoretical literature. Since the field is expanding rapidly, it is impossible in limited space to describe adequately all of the key papers. Therefore, for more comprehensive coverage of the literature describing the mathematical and computational modelling of early avascular and vascular tumour growth, we direct the interested reader to the excellent reviews by Araujo and McElwain [5], Roose et al. [72], Tracqui [79], Lowengrub et al. [55] and Preziosi [70] (and references therein).

Most models of vascular tumour growth are based upon simpler models of avascular tumour growth. Theoretical investigations focussed initially on developing models of avascular tumour growth for several reasons: fewer processes are involved, data from in vitro experiments is readily available and the tumours are often geometrically "simple", growing either as radially-symmetric aggregates in $3 \mathrm{D}$ or as circular monolayers in 2D [27, 49]. Further, what is typically of interest is an equilibrium state for which cell proliferation in nutrient-rich regions balances cell death in nutrient-starved regions. The resulting models of avascular tumour growth can be decomposed into two categories: continuum models comprising systems of partial differential equations [35, 36], and discrete models that treat each cell as a distinct entity which interacts with its neighbours $[22,58]$. Increasingly, the continuum and discrete approaches are combined to give hybrid models which couple processes acting at different spatial scales. For example, angiogenesis (the formation of new blood vessels), which must occur if the tumour is to become vascularised, is often modelled as a discrete 
process in which individual endothelial cells move in response to chemical gradients which are specified by coupled reaction-diffusion equations (see, for example, [19, 57, 65, 68]). An excellent review of these approaches can be found in [72].

The earliest spatially-resolved models of vascular tumour growth were formulated as systems of coupled reaction-advection-diffusion equations. For example, Orme and Chaplain's [62] model of the evolution of tumour cell and capillary densities included homogeneous diffusion of both species, taxis of tumour cells up a gradient of capillary vessels and source terms representing both proliferation and death of tumour cells and blood vessels. The accompanying mathematical and numerical analysis focussed on one-dimensional, radially-symmetric growth and showed that the system could generate a well-developed tumour, with a central necrotic core surrounded by an annulus containing blood vessels and proliferating tumour cells.

In [12], Breward, Byrne and Lewis used a multiphase approach to derive a reactionadvection-diffusion model to describe the co-evolution of live and dead tumour cells in a micro-region surrounding a blood vessel which acts as a fixed source of oxygen and is localised on the boundary of the domain. The velocity field driving cell movement was obtained by assuming that the cells' velocity was proportional to the local pressure gradient and appealing to Darcy's Law. In later work, Jackson and Byrne [45] extended this multicomponent reaction-advection-diffusion framework to investigate the impact of the vasculature on tumour drug resistance.

Hogea et al. [44] explicitly incorporated blood vessels into a multicomponent reactionadvection-diffusion model, which relates the behaviour of healthy cells, tumour cells and capillaries to the supply of nutrient and angiogenic factor. Their work also exemplifies a standard procedure, in which the computational domain is divided into distinct regions (here, inside and outside the tumour), each characterised by a different system of equations, and the interfaces between them tracked explicitly. In [44] the tumour boundary is tracked using a level set method. Macklin et al. employed a similar reaction-advection-diffusion approach to develop a hybrid model [57] in which the transport of chemical species is represented using a continuum model, and coupled with a discrete simulation of the growth of new blood vessels. In this model, the cells are assumed to move in response to the local pressure gradient (Darcy's law) and, via haptotaxis, the spatial gradient of a non-diffusible chemical such as laminin or fibronectin which is bound to the extracellular tissue matrix. Hybrid approaches of this type are becoming increasingly popular because they allow different biological processes to be represented at scales that are appropriate to a particular simulation, e.g. cells may be represented as individual entities at one scale while diffusible species are treated as continua. The hybrid/multiscale nature of [57] derives from the treatment of the vasculature as a network of discrete branches, rather than as a separate phase in the continuum framework. A similar hybrid structure was proposed by Zheng et al. [83] but the velocity field for all of their cells was governed by a Darcy-Stokes law and the computation was carried out by 
combining an adaptive finite element approximation with a level set approach for tracking the tumour boundary.

In separate work, Alarcón, Byrne and Maini have developed an alternative multiscale model by embedding a vascular network within a discrete population of healthy and cancerous cells, and used their model to study the effects of blood flow and vascular remodelling on the evolution of the two cell populations $[1,2,3]$. The model was extended in [65], to account for changes in the morphology of the vascular network associated with angiogenesis and vessel regression, and in [69], to investigate three-dimensional behaviour and boundary effects. More recent work involves using the model to study the efficacy of treatment strategies which combine standard chemotherapeutic approaches with novel ones. The new treatments involve genetically engineering macrophages to deliver cytotoxic drugs to hypoxic tumour regions and exploit the tendency of macrophages to localise in such low oxygen regions [66].

Gevertz and Torquato $[33,34]$ proposed a similar hybrid structure but used a biochemical model to account for subcellular signalling within the endothelial cells that comprise the blood vessels. A similar framework was implemented by Billy et al. [11], although they modelled the endothelial cells with a reaction-advection-diffusion equation.

A series of papers $[9,20,28,82]$ chart the development of an alternative approach to multicomponent modelling of vascular tumour growth. Here the constitutive laws, used to define the mass fluxes caused by mechanical interactions between the cells and the velocities of each component, are derived from thermodynamic principles applied to the free energy of each component. Models of this type treat tumour cells, host cells and extracellular water as separate components. The tumour boundary is not explicitly tracked: instead, Cahn-Hilliard-type equations provide a diffuse interface which must be captured on the computational mesh. A hybrid version of this approach has also been developed, in which the reaction-advection-diffusion equations governing the behaviour of the cells and extracellular fluid are coupled to a discrete description of the evolving vascular network [28, 29]. This work also makes a significant contribution in terms of computational modelling. The majority of computational simulations of continuum representations of tumour growth are carried out using simple finite difference schemes on uniform, structured, meshes. In order to carry out two- and three-dimensional simulations in which the diffuse interface is accurately resolved, adaptive meshing and multigrid algorithms have been implemented [20, 81] to provide efficient simulation software capable of modelling large-scale problems.

As noted in [72], the models mentioned above typically assume that all components or phases move with the same velocity. In order to relax this constraint, force balances can be applied to each phase, with inter-phase momentum transfer taken into consideration and appropriate constitutive laws used to characterise the material properties of each phase. Such multiphase models provide the foundations for our work, which builds on the models of avascular and vascular tumour growth proposed by Byrne et al. [15] and Breward, Byrne and Lewis $[13,14]$. The original two-phase model of avascular tumour growth [13, 15] decomposes 
the tissue into a tumour cell phase (treated as a viscous liquid) and an extracellular fluid phase (an inviscid liquid) and is derived by appealing to the principles of conservation of mass and momentum for incompressible, inertialess fluids. The two phases interact via exchange of mass, through oxygen-regulated cell birth and death, and exchanged momentum, through inter-phase drag. In [14], the reaction-diffusion equation for oxygen is superseded by a third phase for the blood vessels so that the effects of angiogenesis and vessel occlusion can be incorporated. Despite restricting attention to one-dimensional mathematical analysis and numerical simulations, the latter presented on uniform meshes which expand as the tumour grows, the authors demonstrated that these simple multiphase models can reproduce many of the characteristic features associated with tumour growth, including the development of a necrotic core behind an outward-moving rim of proliferating tumour cells.

Similar principles have been used to develop multiphase models to study tumour capsule formation $[46,56]$, the impact of external mechanical loading on avascular tumour growth [17] and the development of tumour cords and fibrosis [71, 78], cell migration due to random motion and chemotaxis [16] and liver cell aggregation [38]. For example, Roose et al. [73] developed a two-phase model to analyse the stresses generated by spheroid growth when the fluid velocity is governed by Darcy's Law and the tissue stresses described by a linear poroelastic model. Dembo and coauthors have used similar models to study cell division [40], neutrophil migration and phagocytosis [42, 43] and cell motility [41]. Other multiphase models, such as those of Lemon et al. [52, 53], have been specialised for applications in tissue engineering. In such models a fixed, solid, phase is introduced to represent the porous scaffold through which extracellular fluid and cells can flow. Osborne, O'Dea and coauthors $[60,61,63]$ have extended these ideas, in order to study tissue growth in a bioreactor through which viscous fluids are driven by an externally-imposed pressure gradient. In a model of tissue growth, Araujo and McElwain [6, 7] view the tissue as a linear-elastic material which interacts with an inviscid fluid, and investigate the genesis of residual stresses in tissue. Ambrosi and Preziosi [4] place these models in a more general framework which allows for viscoelastic behaviour of the phases and incorporates the effects of cell adhesion.

The model presented in this paper extends the work of Breward, Byrne and Lewis [13, 14] by distinguishing between the blood vessels that perfuse the tissue and the diffusible nutrients that they supply. In this way, the vasculature can influence tissue at a distance from the vessel. We also distinguish between the healthy and tumour cells, allowing interactions between the growing tumour and the healthy tissue (in most existing multiphase models, interactions with the normal tissue surrounding the tumour are neglected). A new computational approach, based on the finite element and finite volume methods, is designed, that can simulate tumour growth in two dimensions on arbitrary computational domains, and that is readily extended to three dimensions. The numerical method is inherently mass-conservative and uses no artificial stabilisation techniques. It differs from the two-dimensional computational approach of Osborne and Whiteley [64] in both its treatment of the mass balance 
equations and in allowing regions where the phase volume fraction of tumour cells is zero, including tissue "outside" the tumour. The interface between the cancerous and healthy tissue is captured in the form of a slightly diffuse moving front rather than a sharp interface which must be tracked explicitly. Osborne and Whiteley [64] treat this interface as a moving boundary of their computational domain when simulating avascular tumour growth, a potential difficulty for vascular tumours where the boundary shape is typically less constrained. The inclusion of healthy cells in the underlying mathematical model also allows more control of the properties of the surrounding tissue.

The remainder of the paper is organised as follows. In Section 2 we present our fourphase model for healthy cells, tumour cells, blood vessels and extracellular material. The numerical algorithm used to approximate the model (a hybrid finite volume/finite element approach on unstructured triangular meshes in two space dimensions) is outlined in Section 3 and numerical results presented in Section 4 illustrate the behaviour of the model and the versatility of the numerical method. We show that the model readily reproduces the characteristic pattern of a necrotic core developing behind a proliferating rim and investigate the dependence of the tumour growth on various model parameters. The paper concludes in Section 5 with a discussion of our results and suggestions for further research.

\section{Model Development}

In this section, we introduce a multiphase model of vascular tumour growth. Following $[13,14]$ we assume that the tissue comprises four interacting phases, for normal/healthy cells, cancer cells, blood vessels and extracellular material. We denote their volume fractions, velocities and pressures respectively by $\theta_{i}, \vec{u}_{i}$ and $p_{i}(i=1, \ldots, 4)$ and derive equations for their evolution by applying to each phase the principles of mass and momentum balance with appropriate constitutive assumptions. We also consider a single, diffusible species $(c)$, such as oxygen or glucose, which is supplied by the blood vessels and acts as a source of nutrients for the normal and cancerous cells. We denote by $\Omega$ the domain in which the equations hold and by $\Gamma$ its boundary.

\subsection{The Mass Balance Equations}

All phases are assumed to have the same (constant) density, so mass conservation supplies

$$
\frac{\partial \theta_{i}}{\partial t}+\vec{\nabla} \cdot\left(\theta_{i} \vec{u}_{i}\right)=q_{i}, \quad i=1, \ldots, 4
$$

In Equation (1), $\theta_{i}$ are volume fractions, $\vec{u}_{i}$ are phase velocities and $q_{i}$ are source/sink terms representing the mass transfer between the different phases $(i=1, \ldots, 4)$ that is associated with processes such as cell birth and death, angiogenesis and vessel occlusion ( $c f$. [14]). 
Following [14], we make the further assumption that the tissue has no voids so that

$$
\sum_{i=1}^{4} \theta_{i}=1
$$

We also require that total mass is conserved so that any volume lost from one phase, via a sink term, will be balanced by an equal volume increase in another phase (and vice versa). Under this assumption we have that

$$
\sum_{i=1}^{4} q_{i}=0 .
$$

In the subsections that follow, we introduce the source and sink terms associated with each phase.

\subsubsection{The Normal and Cancer Cell Phases $\left(\theta_{1}, \theta_{2}\right)$}

We assume that the volume fractions of the healthy and tumour cells $\left(\theta_{1}, \theta_{2}\right.$, respectively) increase through cell proliferation and decrease due to cell death, with extracellular material $\left(\theta_{4}\right)$ supplying the material needed to drive cell growth and proliferation, and both rates being regulated by the local nutrient concentration. The proliferation rates are assumed to be monotonically increasing and saturating functions of $c$, increasing from zero when $c=0$, to a finite maximum $k_{1, i}$ in the limit as $c \rightarrow \infty$. The cell death rate for each phase is assumed to be proportional to the volume fraction of that phase and to decrease monotonically with $c$ from a maximum value of $k_{2, i} c_{c_{1}} / c_{c_{2}}$ when $c=0$ (i.e. maximal necrosis under hypoxia) to a minimum or basal rate of $k_{2, i}$ as $c \rightarrow \infty$. Combining the above assumptions we obtain mass balance source terms for the healthy and cancer cells of the form

$$
\begin{aligned}
& q_{1}=\underbrace{k_{1,1} \theta_{1} \theta_{4}\left(\frac{c}{c_{p}+c}\right)}_{\text {cell birth }} \underbrace{-k_{2,1} \theta_{1}\left(\frac{c_{c_{1}}+c}{c_{c_{2}}+c}\right)}_{\text {cell death }} \\
& q_{2}=\underbrace{k_{1,2} \theta_{2} \theta_{4}\left(\frac{c}{c_{p}+c}\right)}_{\text {cell birth }} \underbrace{-k_{2,2} \theta_{2}\left(\frac{c_{c_{1}}+c}{c_{c_{2}}+c}\right)}_{\text {cell death }} .
\end{aligned}
$$

We remark that the above expressions for $q_{1}$ and $q_{2}$ include four rate constants $\left(k_{1,1}, k_{2,1}\right.$, $\left.k_{1,2}, k_{2,2}\right)$ and three nutrient concentration parameters $\left(c_{p}, c_{c_{1}}, c_{c_{2}}\right)$, two of which $\left(c_{p}, c_{c_{2}}\right)$ represent values at which the relevant rates are half-maximal. In Equations (4), the only distinction made between healthy cells and tumour cells is in the magnitudes of the rate constants, $k_{i, j}$. Since tumour cells typically proliferate more rapidly and are less likely to die under nutrient-poor conditions than normal cells, we suppose that $k_{1,2} \geq k_{1,1}$ and $k_{2,2} \leq k_{2,1}$. In order to ensure that the death rate increases as the nutrient concentration decreases, we assume further that $c_{c_{1}}>c_{c_{2}}$. Finally, for simplicity, we have assumed that the threshold nutrient concentrations, $c_{p}, c_{c_{1}}$ and $c_{c_{2}}$, are identical for normal and cancer cells. Sketches showing how the birth and death rates depend on the nutrient concentration $c$ are presented in Figure 1. 

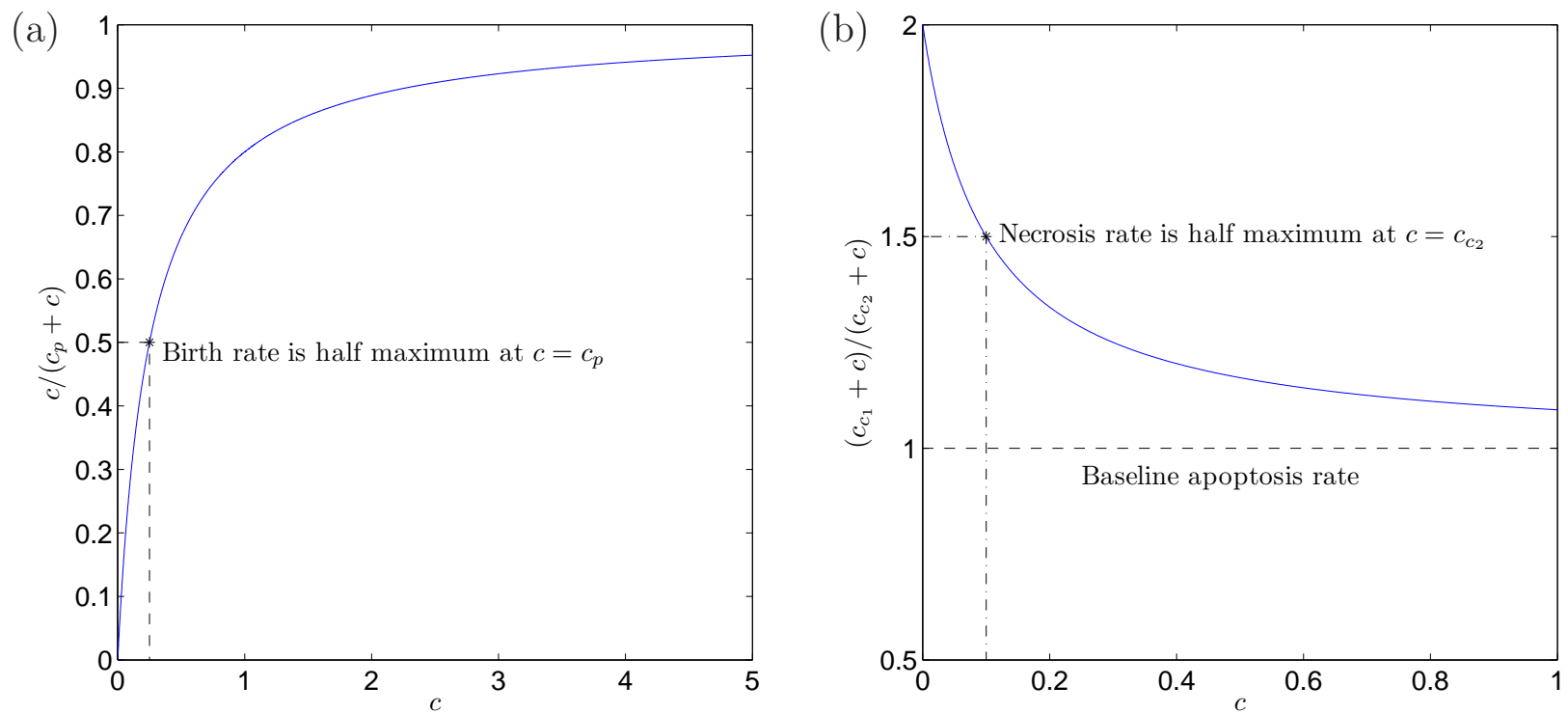

Figure 1: Illustrative sketches showing the dependence of (a) cell birth rate and (b) cell death rate on the nutrient concentration, $c$. Parameter values: $c_{p}=0.25, c_{c_{1}}=0.2, c_{c_{2}}=0.1$. Scaling by the constant multipliers $k_{1, i}$ and $k_{2, i}$ is ignored here.

\subsubsection{Blood Vessel Phase $\left(\theta_{3}\right)$}

The volume fraction of blood vessels increases through angiogenesis and decreases due to occlusion. Accordingly we suppose that vessels are removed or pruned from the vasculature if the pressure exerted on them by the surrounding cells $\left(\theta_{1} p_{1}+\theta_{2} p_{2}\right)$ exceeds the critical value $p_{\text {crit }}$ (in practice, the vessels first become occluded, then their blood flow declines, reducing the wall shear stress that they experience and ultimately leading to their regression). Hence the pruned vessel mass acts as a source in the mass balance for the extracellular material (see Equation (7). The rate of new vessel formation is assumed to be proportional to the volume fraction of blood vessels $\left(\theta_{3}\right)$ and the total volume fraction of the cells $\left(\theta_{1}+\theta_{2}\right)$, the cells acting as a source of angiogenic factor (in the absence of a separate diffusible species such as VEGF). It is assumed that vessel growth is inhibited when there is too little extracellular material to form new vessels, and that the growth rate increases monotonically towards a bounded maximum as $\theta_{4}$ increases. We account for the dependence of the rate of angiogenesis on the local nutrient/oxygen concentration by supposing further that vessel growth is inhibited when nutrient levels are either low or high, and maximal for intermediate values of $c$ (in practice, the cells only express the angiogenic factors, such as VEGF, that stimulate vessel growth when nutrient levels are between these threshold values). Healthy cells and tumour cells are assumed to act identically as sources of angiogenic factor.

Combining the above assumptions we obtain the following expression for $q_{3}$, the mass balance source term in Equation (1):

$$
q_{3}=\underbrace{-k_{3} \theta_{3} \mathcal{H}\left(\theta_{1} p_{1}+\theta_{2} p_{2}-p_{\text {crit }}, \epsilon_{3}\right)}_{\text {occlusion }} \underbrace{+k_{4}\left(\theta_{1}+\theta_{2}\right) \theta_{3}\left(\frac{\theta_{4}}{\varepsilon+\theta_{4}}\right)\left(\frac{c}{\left(c_{a}+c\right)^{2}}\right)}_{\text {angiogenesis }},
$$


where $k_{3}$ and $k_{4}$ are rate constants, $\varepsilon$ is the blood vessel volume fraction at which the angiogenesis rate is half-maximal, $c_{a}$ is the nutrient concentration at which the rate of angiogenesis is maximal, and

$$
\mathcal{H}(p, \epsilon)=\frac{1}{2}\left(1+\tanh \frac{p}{\epsilon}\right), \quad \epsilon \ll 1,
$$

is a smooth approximation to the Heaviside step function (see Figure 2).
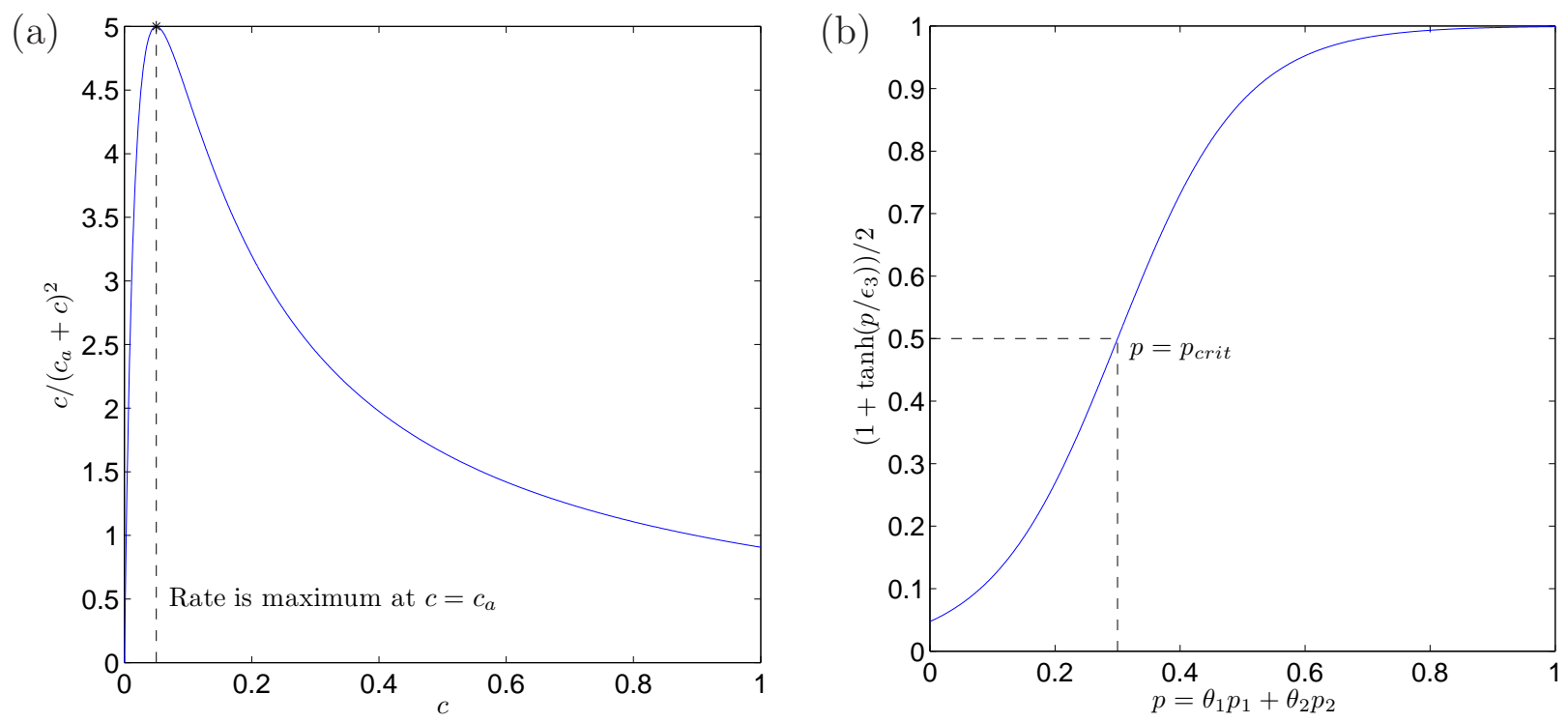

Figure 2: Illustrative sketches showing the dependence of (a) vessel occlusion rate on cell partial pressures for $p_{\text {crit }}=0.3$ and $\epsilon_{3}=0.2$ and (b) angiogenesis rate on nutrient concentration for $c_{a}=0.05$. Scaling by the constant multipliers $k_{3}$ and $k_{4}$ is ignored here.

\subsubsection{Extracellular Material Phase $\left(\theta_{4}\right)$}

The extracellular material is a simplistic representation of a phase which includes both the additional material required to create volume in the other phases, i.e. during mitosis and angiogenesis, and the material that remains when volume from another phase is lost, i.e. during apoptosis, necrosis and vessel occlusion. The system is assumed to be closed, with no external replenishment of resources except that related to balancing fluxes across the boundary of the computational domain. It follows that the mass source term in (1) for the 
extracellular material phase takes the form

$$
\begin{aligned}
q_{4}= & -q_{1}-q_{2}-q_{3} \\
= & \underbrace{-k_{1,1} \theta_{1} \theta_{4}\left(\frac{c}{c_{p}+c}\right)}_{\text {healthy cell birth }} \underbrace{+k_{2,1} \theta_{1}\left(\frac{c_{c_{1}}+c}{c_{c 2}+c}\right)}_{\text {healthy cell death }} \\
& \underbrace{-k_{1,2} \theta_{2} \theta_{4}\left(\frac{c}{c_{p}+c}\right)}_{\text {tumour cell birth }} \underbrace{+k_{2,2} \theta_{2}\left(\frac{c_{c_{1}}+c}{c_{c_{2}}+c}\right)}_{\text {tumour cell death }} \\
& \underbrace{+k_{3} \theta_{3} \mathcal{H}\left(\theta_{1} p_{1}+\theta_{2} p_{2}\right.}_{\text {vessel occlusion }}-p_{\text {crit }, \epsilon_{3}} \underbrace{-k_{4}\left(\theta_{1}+\theta_{2}\right) \theta_{3}\left(\frac{\theta_{4}}{\varepsilon+\theta_{4}}\right)\left(\frac{c}{\left(c_{a}+c\right)^{2}}\right)}_{\text {angiogenesis }} .
\end{aligned}
$$

Since the extracellular material is essentially a passive medium which provides material for, or accepts material from, processes relating to the other phases, its volume fraction is calculated in the following using the no-voids condition (2) instead of its mass balance equation. In other words, the equation used to update the ECM phase volume fraction is

$$
\theta_{4}=1-\theta_{1}-\theta_{2}-\theta_{3} .
$$

This ensures that conservation of mass is preserved when the system is discretised.

\subsubsection{Initial and Boundary Conditions}

In order to close the mass balance equations (1), $\theta_{i}$ is prescribed for each phase and for all $t \geq$

0 on the corresponding inflow section of the boundary, $\Gamma_{i}^{\text {Inflow }}$. The inflow sections are defined to be those parts of $\Gamma$ on which $\vec{u}_{i} \cdot \hat{\vec{n}}<0$, where $\hat{\vec{n}}$ is the outward-pointing unit normal to the boundary. Since the mass balance equations are hyperbolic, with information propagating along characteristic curves, no condition is required on the remainder of the boundary. Initial conditions must also be provided on the whole of $\Omega$ for these time-dependent equations.

\subsection{Momentum Balance}

Assuming that the Reynolds number of the flow is low enough to neglect inertial terms, conservation of momentum can be written in the form

$$
\vec{\nabla} \cdot\left(\theta_{i} \boldsymbol{\sigma}_{i}\right)+\vec{F}_{i}=0, \quad i=1, \ldots, N_{p},
$$

in which $\boldsymbol{\sigma}_{i}$ are the stresses in each of the individual phases and $\vec{F}_{i}$ are the corresponding momentum sources and sinks. As will be demonstrated, these equations are not, on their own, enough to determine the velocities $\vec{u}_{i}$ which govern the flow in the mass balance equations (1). They must be supplemented by a continuity equation for the phase mixture, which immediately follows from summing (1) to give

$$
\sum_{i=1}^{N_{p}} \vec{\nabla} \cdot\left(\theta_{i} \vec{u}_{i}\right)=0,
$$


and appropriately defined constitutive relations for phase pressures.

\subsubsection{Constitutive Assumptions}

The mechanical behaviour of the tissue is modelled by assuming that each phase behaves like a viscous fluid, and has associated with it a stress tensor of the form

$$
\boldsymbol{\sigma}_{i}=-p_{i} \mathbf{I}+\mu_{i}\left(\vec{\nabla} \vec{u}_{i}+\left(\vec{\nabla} \vec{u}_{i}\right)^{\mathrm{T}}\right)+\lambda_{i}\left(\vec{\nabla} \cdot \vec{u}_{i}\right) \mathbf{I}, \quad i=1, \ldots, N_{p},
$$

in which $p_{i}$ are the phase pressures, and $\mu_{i}, \lambda_{i}$ are the dynamic shear and bulk viscosities, respectively. In this work it is assumed that the phases are in local thermodynamic equilib-

rium, so $\lambda_{i}=-\frac{2}{3} \mu_{i}$ throughout. The effects of pressure are also included in the momentum sources in (9), along with terms representing inter-phase drag. This leads to

$$
\vec{F}_{i}=p_{i} \mathbf{I} \vec{\nabla} \theta_{i}+\sum_{j=1, j \neq i}^{N_{p}} d_{i j} \theta_{i} \theta_{j}\left(\vec{u}_{j}-\vec{u}_{i}\right), \quad i=1, \ldots, N_{p},
$$

in which $d_{i j}$ is the drag coefficient associated with the relative movement between phases $i$ and $j$.

In order to close the model, algebraic relationships between the different phase pressures are prescribed. While several alternatives have been proposed [17], the expressions employed here are based on those used in $[13,14]$. First, it is assumed that the pressure in the blood vessel phase is constant, so that $p_{3}=p_{3}^{*}$, where $p_{3}^{*}$ is the externally-imposed pressure in the vasculature. Second, the healthy and tumour cells are assumed to act like isotropic fluids, with additional cell-cell interactions, so that

$$
p_{1}=p_{2}=p_{4}+\Sigma(\theta),
$$

where $\theta=\theta_{1}+\theta_{2}$ is the total cell volume fraction. For simplicity, the healthy cells and the tumour cells are assumed to interact with each other in precisely the same manner, though it would be interesting to examine the effects of modifying their behaviour. The cells have a natural density $\theta^{*}$, below which they are so sparsely distributed that they experience no stress and do not interact. For $\theta=\theta_{1}+\theta_{2}>\theta^{*}$ the cells move to reduce their stress. Accordingly, the functional form used to define $\Sigma(\theta)$ is $[13,14]$

$$
\Sigma(\theta)=\left\{\begin{array}{cl}
\frac{\Lambda\left(\theta-\theta^{*}\right)}{(1-\theta)^{2}} & \text { if } \theta \geq \theta^{*} \\
0 & \text { if } \theta<\theta^{*}
\end{array}\right.
$$

where the tension constant, $\Lambda$, is a measure of the cells' affinity for their natural density. This relationship is illustrated in Figure 3.

\subsubsection{Boundary Conditions}

The system which couples the momentum balance equations (9) with the incompressibility constraint (10) and the pressure relations (13) requires additional conditions on the boundary of the domain $\Gamma$ before the profiles of $\vec{u}_{i}$ and $p_{i}$ can be found. 


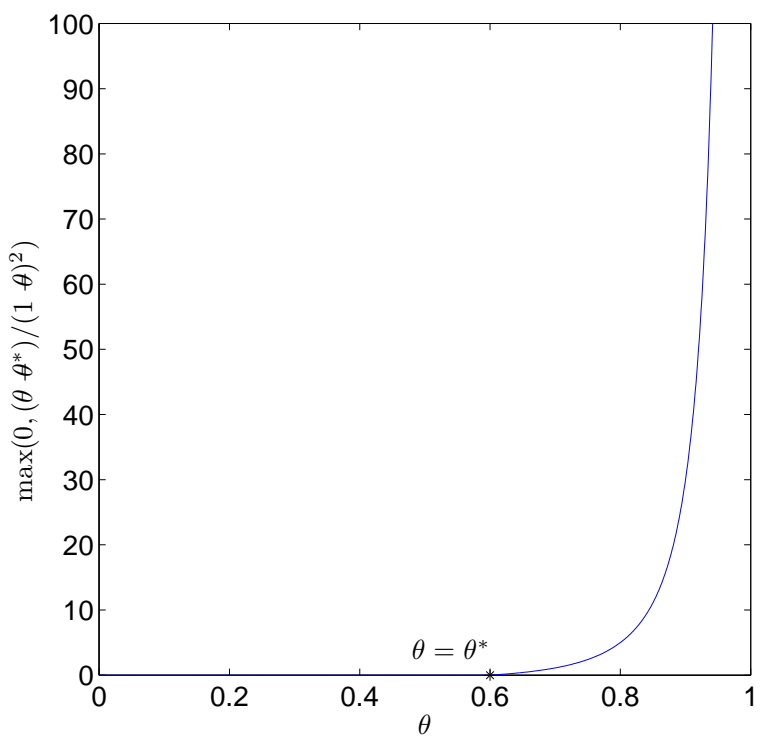

Figure 3: Illustrative sketch showing the dependence of the cell-induced pressures, $\Sigma(\theta) / \Lambda$, on the total cell phase volume fraction $\theta$ when $\theta^{*}=0.6$.

- For sections of the boundary which represent solid obstacles, $\vec{u}_{i}=0$ is imposed for $i=1, \ldots, N_{p}$. If the whole of $\Gamma$ is a solid obstacle, $p_{i}$ must also be specified at one point in the domain in order to obtain a unique solution.

- For the remaining sections of the boundary, the normal stress $\boldsymbol{\sigma}_{i} \cdot \hat{\vec{n}}$ is specified, where $\hat{\vec{n}}$ is the outward-pointing unit normal to $\Gamma$. Note though that it is not possible to specify the normal stress around the whole boundary for all phases: $\vec{u}_{i}$ must be specified along some section of the boundary for at least one phase in order to obtain a unique solution.

\subsection{Diffusible Species}

The contribution of the diffusible species to the overall volume is assumed to be negligible and their transport taken to be diffusion-dominated, and to equilibrate with the reaction processes on timescales which are considerably shorter (i.e. minutes) than those associated with changes in the phase volume fractions (i.e. weeks or months). Under these assumptions [12], the diffusible species are governed by the quasi-steady reaction-diffusion equations of the form

$$
D_{j} \vec{\nabla}^{2} c_{j}+q_{j}=0, \quad j=1, \ldots, N_{d},
$$

in which $c_{j}\left(j=1, \ldots, N_{d}\right)$ denote the concentrations of the diffusible species. The diffusion coefficients $D_{j}$ are assumed constant and the $q_{j}$ represent the net source terms (i.e. sources - sinks) associated with each species. 


\subsubsection{Nutrient/Oxygen}

The single diffusible species used here, denoted simply by $c$, represents nutrient supplied by the vasculature and consumed by both normal and cancer cells, providing them with the energy that they need, not only to remain alive, but also to proliferate. For simplicity, the rate at which the two cell types consume nutrient in order to carry out normal, baseline, activities is assumed to be proportional to the nutrient concentration and their respective volume fractions. Additional nutrient is consumed by the normal and cancer cells at rates which are proportional to their proliferation rates (see Equations (4)).

Combining these processes leads to a source term $q_{c}$ in the nutrient diffusion equation (15) which takes the form

$$
\begin{aligned}
q_{c}=\underbrace{k_{5} \theta_{3}\left(c_{v}-c\right)}_{\text {replenishment }} \underbrace{-k_{6,1} \theta_{1} c-k_{6,2} \theta_{2} c}_{\text {baseline consumption }} \\
\underbrace{-k_{7,1} \theta_{1} \theta_{4}\left(\frac{c}{c_{p}+c}\right)-k_{7,2} \theta_{2} \theta_{4}\left(\frac{c}{c_{p}+c}\right)}_{\text {consumption due to cell birth }} .
\end{aligned}
$$

This expression contains five rate constants $\left(k_{5}, k_{6,1}, k_{6,2}, k_{7,1}\right.$ and $\left.k_{7,2}\right)$, and it is assumed that $k_{7,1} / k_{7,2}=k_{1,1} / k_{1,2}$ for consistency with the cell birth terms in (4). The parameter $c_{p}$ is identical to that used in the cell phase source terms of Equation (4), while $c_{v}$ represents the assumed constant concentration of the nutrient within the blood vessels. The model could easily be extended to account for other blood-borne species, such as glucose or drugs.

\subsubsection{Boundary Conditions}

Equations (15) for the distribution of the diffusible species, $c_{j}$, can be closed by prescribing either Dirichlet or Neumann boundary conditions. For the former, $c_{j}$ is specified on the

domain boundary $\Gamma$, while for the latter, $\vec{\nabla} c_{j} \cdot \hat{\vec{n}}$ is specified, where $\hat{\vec{n}}$ is the outward-pointing unit normal to $\Gamma$.

\subsection{Nondimensionalisation}

Before presenting simulation results, it is convenient to recast the model in dimensionless form. Since the phase volume fractions $\theta_{1}, \theta_{2}, \theta_{3}$ and $\theta_{4}$ are dimensionless (by definition), we scale only the independent variables, $\vec{x}$ and $t$, the phase velocities $\vec{u}_{i}$, the pressures $p_{i}$, and the nutrient concentration $c$. This is done in the following way:

$$
t=\frac{t^{\prime}}{k_{1,1}}, \quad \vec{x}=L_{0} \vec{x}^{\prime}, \quad \vec{u}_{i}=L_{0} k_{1,1} \vec{u}_{i}^{\prime}, \quad p_{i}=\Lambda p_{i}^{\prime}, \quad c=c_{v} c^{\prime},
$$

where primes denote dimensionless variables. The birth rate parameter for healthy cells, $k_{1,1}$, is used to scale time, $L_{0}$ is a typical length scale (taken later to be the initial radius of the tumour seeded in the healthy tissue), $\Lambda$ is the cell-cell interaction tension constant (see Equation (14)) and $c_{v}$ is the nutrient concentration in the blood vessels (see Equation (16)). 


\subsubsection{Mass Balance Equations}

Substituting from Equations (17) in Equations (1) leads to the following evolution equations for $\theta_{1}, \theta_{2}$ and $\theta_{3}$, the phase volume fractions of the cells and blood vessels:

$$
\begin{aligned}
\frac{\partial \theta_{1}}{\partial t^{\prime}}+\vec{\nabla}^{\prime} \cdot\left(\theta_{1} \vec{u}_{1}^{\prime}\right) & =\underbrace{\theta_{1} \theta_{4}\left(\frac{c^{\prime}}{c_{p}^{*}+c^{\prime}}\right)}_{\text {cell birth }} \underbrace{-k_{2,1}^{*} \theta_{1}\left(\frac{c_{c_{1}}^{*}+c^{\prime}}{c_{c_{2}}^{*}+c^{\prime}}\right)}_{\text {cell death }}, \\
\frac{\partial \theta_{2}}{\partial t^{\prime}}+\vec{\nabla}^{\prime} \cdot\left(\theta_{2} \vec{u}_{2}^{\prime}\right) & =\underbrace{k_{1,2}^{*} \theta_{2} \theta_{4}\left(\frac{c^{\prime}}{c_{p}^{*}+c^{\prime}}\right)}_{\text {cell birth }} \underbrace{-k_{2,2}^{*} \theta_{2}\left(\frac{c_{c_{1}}^{*}+c^{\prime}}{c_{c_{2}}^{*}+c^{\prime}}\right)}_{\text {cell death }}, \\
\frac{\partial \theta_{3}}{\partial t^{\prime}}+\vec{\nabla}^{\prime} \cdot\left(\theta_{3} \vec{u}_{3}^{\prime}\right) & =\underbrace{-k_{3}^{*} \theta_{3} \mathcal{H}\left(\theta_{1} p_{1}^{\prime}+\theta_{2} p_{2}^{\prime}-p_{c r i t}^{*} \epsilon_{3}^{*}\right)}_{\text {occlusion }}
\end{aligned}
$$

in which

$$
\begin{gathered}
k_{2,1}^{*}=\frac{k_{2,1}}{k_{1,1}}, \quad k_{1,2}^{*}=\frac{k_{1,2}}{k_{1,1}}, \quad k_{2,2}^{*}=\frac{k_{2,2}}{k_{1,1}}, \quad k_{3}^{*}=\frac{k_{3}}{k_{1,1}}, \quad k_{4}^{*}=\frac{k_{4}}{c_{v} k_{1,1}}, \\
c_{p}^{*}=\frac{c_{p}}{c_{v}}, \quad c_{a}^{*}=\frac{c_{a}}{c_{v}}, \quad c_{c_{1}}^{*}=\frac{c_{c_{1}}}{c_{v}}, \quad c_{c_{2}}^{*}=\frac{c_{c_{2}}}{c_{v}}, \\
p_{\text {crit }}^{*}=\frac{p_{\text {crit }}}{\Lambda}, \quad \epsilon_{3}^{*}=\frac{\epsilon_{3}}{\Lambda} .
\end{gathered}
$$

As mentioned in Section 2.1.3, the phase volume fraction of extracellular material is calculated from the no-voids condition, which remains unchanged by the nondimensionalisation, i.e. $\theta_{4}=1-\theta_{1}-\theta_{2}-\theta_{3}$. When imposing boundary conditions, $\theta_{i}$ is prescribed for each phase on $\Gamma_{i}^{\text {Inflow }}$, the sections of $\Gamma$ for which $\vec{u}_{i}^{\prime} \cdot \hat{\vec{n}}^{\prime}<0$, where $\hat{\vec{n}}^{\prime}$ is the outward-pointing unit normal to the boundary.

\subsubsection{Momentum Balance Equations}

Equations (9)-(13) transform to give the following equations for the dimensionless phase velocities $\vec{u}_{i}^{\prime}$ and phase pressures $p_{i}^{\prime}$ :

$$
\begin{aligned}
\sum_{j \neq i} d_{i j}^{*} \theta_{i} \theta_{j}\left(\vec{u}_{j}^{\prime}-\vec{u}_{i}^{\prime}\right)-\theta_{i} \vec{\nabla}^{\prime} \cdot\left(\Lambda^{*} p_{i}^{\prime} \mathbf{I}\right) & \\
+\vec{\nabla}^{\prime} \cdot\left[\theta_{i}\left[\mu_{i}^{*}\left(\vec{\nabla}^{\prime} \vec{u}_{i}^{\prime}+\left(\vec{\nabla}^{\prime} \vec{u}_{i}^{\prime}\right)^{\mathrm{T}}\right)+\lambda_{i}^{*}\left(\vec{\nabla}^{\prime} \cdot \vec{u}_{i}^{\prime}\right) \mathbf{I}\right]\right] & =0, \quad i, j=1,2,3,4, \\
\sum_{i=1}^{4} \vec{\nabla}^{\prime} \cdot\left(\theta_{i} \vec{u}_{i}^{\prime}\right) & =0, \\
p_{1}^{\prime}=p_{2}^{\prime}=p_{4}^{\prime}+\Sigma^{\prime}(\theta), & p_{3}^{\prime}=\frac{p_{3}^{*}}{\Lambda},
\end{aligned}
$$


in which $d_{i j}=d_{j i}$,

$$
d_{i j}^{*}=\frac{d_{i j}}{d_{12}}, \quad \Lambda^{*}=\frac{\Lambda}{d_{12} k_{1,1} L_{0}{ }^{2}}, \quad \mu_{i}^{*}=\frac{\mu_{i}}{d_{12} L_{0}{ }^{2}}, \quad \lambda_{i}^{*}=\frac{\lambda_{i}}{d_{12} L_{0}{ }^{2}},
$$

where $i, j=1, \ldots, 4$ and $j \neq i$, and

$$
\Sigma^{\prime}(\theta)=\left\{\begin{array}{cl}
\frac{\left(\theta-\theta^{*}\right)}{(1-\theta)^{2}} & \text { if } \quad \theta \geq \theta^{*} \\
0 & \text { if } \theta<\theta^{*}
\end{array}\right.
$$

with $\theta=\theta_{1}+\theta_{2}$. The simple algebraic relationships between the pressures defined in Equation (20) can be used to rewrite the remaining equations in terms of $\vec{u}_{i}^{\prime}$ and $p_{4}^{\prime}$ only. On $\Gamma$ we impose either $\vec{u}_{i}^{\prime}=0 i=1, \ldots, 4$ (at a solid obstacle), or we prescribe $\boldsymbol{\sigma}_{i}^{\prime} \cdot \hat{\vec{n}}$, as described in Section 2.2.2.

\subsubsection{Reaction-Diffusion Equation}

Finally, the nutrient concentration equation (15), with source terms (16), becomes

$$
\begin{aligned}
D_{c}^{*} \nabla^{\prime 2} c^{\prime}= & \underbrace{\theta_{3}\left(1-c^{\prime}\right)}_{\text {replenishment }} \underbrace{-k_{6,1}^{*} \theta_{1} c^{\prime}-k_{6,2}^{*} \theta_{2} c^{\prime}}_{\text {baseline consumption }} \\
& \underbrace{-k_{7,1}^{*} \theta_{1} \theta_{4}\left(\frac{c^{\prime}}{c_{p}^{*}+c^{\prime}}\right)-k_{7,2}^{*} \theta_{2} \theta_{4}\left(\frac{c^{\prime}}{c_{p}^{*}+c^{\prime}}\right)}_{\text {consumption due to cell birth }},
\end{aligned}
$$

in which

$$
D_{c}^{*}=\frac{D_{c}}{k_{5} L_{0}^{2}}, \quad k_{6,1}^{*}=\frac{k_{6,1}}{k_{5}}, \quad k_{6,2}^{*}=\frac{k_{6,2}}{k_{5}}, \quad k_{7,1}^{*}=\frac{k_{7,1}}{c_{v} k_{5}}, \quad k_{7,2}^{*}=\frac{k_{7,2}}{c_{v} k_{5}},
$$

and $c_{p}^{*}$ is as given in (19). Since this equation does not contain a time-derivative, the timescale used to nondimensionalise it is arbitrary. Here it has been scaled with $k_{5}^{-1}$, the timescale on which nutrient exchanges between the tissue and the blood vessels. On the boundary, either $c^{\prime}$ (Dirichlet conditions) or $\vec{\nabla}^{\prime} c^{\prime} \cdot \hat{\vec{n}}^{\prime}$ (Neumann conditions) can be specified, depending on the details of the problem under investigation. In all cases shown in this paper, $\vec{\nabla}^{\prime} c^{\prime} \cdot \hat{\vec{n}}^{\prime}=0$ is imposed.

\section{The Numerical Algorithm}

The computational simulations presented in $[13,14]$ were carried out in one space dimension using finite differences on a uniform, moving, mesh which grew with the expanding tumour. Only linear reaction terms were considered in the equations governing the diffusible species. Even in multiple space dimensions, finite difference schemes are the preferred method for simulating tumour growth $[3,44,57]$. The boundary of the tumour may be tracked using a level set method $[44,71,83]$, but modelling growth within a complex geometry remains 
challenging. A more natural approach is to use finite element methods on unstructured meshes, which can be tailored to a particular geometry [63, 64, 83].

In this paper we approximate the partial differential equations derived in Section 2 in two space dimensions on unstructured triangular meshes. These are fitted to the problem geometry and provide a framework within which non-uniform and adaptive meshes can be used to improve efficiency. In contrast to [64], where triangular meshes and the same finite element scheme are used to approximate the momentum balance equations, we use a conservative, upwind, finite volume scheme to approximate the hyperbolic mass transport equations and a nonlinear solver for the reaction-diffusion equations. Furthermore, the interface between the growing tumour and the surrounding healthy tissue is captured as a diffuse internal front, and does not need to be tracked explicitly either as an internal interface using a level set method, or as a moving boundary. As a result, it is not necessary to remesh when the (internal) boundary becomes convoluted. Additionally, no artificial stabilisation techniques are used.

The two-dimensional results presented in this paper demonstrate the feasibility of using multidimensional, multiphase models to simulate tumour growth and to investigate how the tumour's spatio-temporal evolution depends on the model parameters.

\subsection{Phase Volume Fractions}

The scalar hyperbolic equations given by (19) are approximated using a standard cell-centre finite volume scheme with forward Euler time-stepping. This involves approximating the integral form of Equations (1),

$$
\int_{\triangle} \frac{\partial \theta_{i}}{\partial t} d \vec{x}+\int_{\triangle} \vec{\nabla} \cdot\left(\theta_{i} \vec{u}_{i}\right) d \vec{x}=\int_{\triangle} q_{i} d \vec{x}, \quad i=1, \ldots, 4,
$$

in which $\triangle$ represents the control volume over which the integration is carried out (a triangle of the computational mesh in this case). The unknowns of the discrete system are cellaverage values of the variable $\theta_{i}$, and each unknown is updated using an equation derived by integrating over the corresponding control volume (mesh triangle). Before the integrals are approximated, the Gauss divergence theorem is applied to the flux integrals in each equation, giving

$$
\int_{\triangle} \frac{\partial \theta_{i}}{\partial t} d \vec{x}+\oint_{\partial \triangle}\left(\theta_{i} \vec{u}_{i}\right) \cdot \hat{\vec{n}} d s=\int_{\triangle} q_{i} d \vec{x}, \quad i=1, \ldots, 4,
$$

where $\partial \triangle$ is the boundary of the control volume $\triangle$, and $\hat{\vec{n}}$ represents the outward-pointing unit normal to this boundary (see Figure 4). In this form it is straightforward to construct approximations which conserve mass, simply by ensuring that the surface integral over an interior mesh edge is evaluated in the same way in both of the adjacent mesh cells. It is also straightforward to impose boundary conditions by evaluating integrals over inflow boundary edges using the specified exterior state ( $c f$. Section 2.1.4). The inflow edges are automatically detected by using an upwind scheme to approximate the fluxes [54, 77] (see Figure 4). 
a)

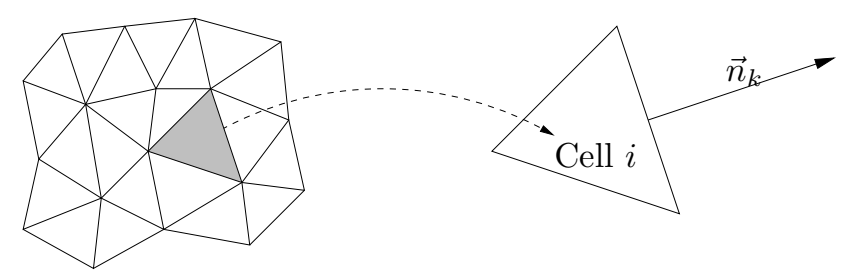

b)

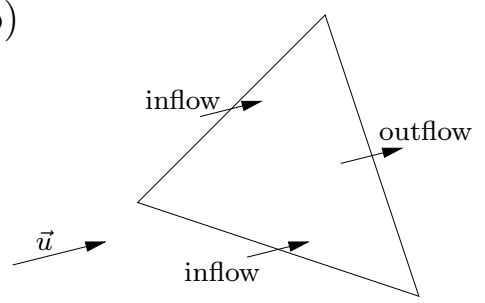

Figure 4: a) A typical triangular mesh cell and its edge normal; b) An illustration of inflow and outflow edges for a triangular mesh cell.

Once the integrals and time derivatives have been approximated using this cell-centred approach, each triangle supplies a discrete equation of the form

$$
\bar{\theta}_{i}^{n+1}=\bar{\theta}_{i}^{n}-\frac{\Delta t}{|\triangle|} \sum_{k=1}^{3}\left(\theta_{i}^{n} \vec{u}_{i}^{n}\right)_{k}^{*} \cdot \vec{n}_{k}+\Delta t\left(q_{i}^{n}\right)^{*}, \quad i=1, \ldots, N_{p},
$$

in which $n$ indexes the time level, $\Delta t$ is the length of the time-step, $|\Delta|$ is the area of the triangular control volume, and $\vec{n}_{k}$ is the outward-pointing normal to the cell edge opposite vertex $k$, scaled by the length of the edge (as illustrated in Figure 4). The asterisks indicate quantities that have been approximated according to the underlying representation of the variable $\theta_{i}$, which is reconstructed within each cell, given knowledge of the cell-average values (denoted here by $\bar{\theta}_{i}$ ) in the nearby cells.

The numerical fluxes $\left(\theta_{i} \vec{u}_{i}\right)_{k}^{*}$ are approximated using a standard upwind approach, with a limited central difference reconstruction of the $\theta_{i}$ [8]. This is a simple multidimensional generalisation of the minmod-limited MUSCL approach [80] which first constructs a linear representation of the variable within each cell, given knowledge of its values in neighbouring cells, and then adjusts it by "limiting" the gradient of the reconstruction to prevent unphysical oscillations appearing in the solution. The scheme is second order accurate in space, except where there are turning points in the solution, where it drops to first order accuracy in order to avoid creating unwanted new extrema. This is important to ensure that the numerical solutions retain the property of the mathematical model that $\theta_{i} \in[0,1]$.

The overall reconstruction is discontinuous across cell edges, so upwinding is used to choose the appropriate value of $\theta_{i}$ at the interface. The value of $\vec{u}_{i}$ at the midpoint of the cell edge (where the numerical flux is nominally evaluated) can be found by interpolation since the velocity field is approximated using a continuous, piecewise polynomial, representation. The values of $\left(q_{i}\right)^{*}$ are found by using a simple quadrature rule to approximate the volume integral in (26) and normalising the result by the cell area $|\triangle|$.

Remark: Mathematically, solving the mass balance equations (1) for all $N_{p}$ phases is equivalent to using (1) to predict the evolution of the first $N_{p}-1$ phases, and the no-voids condition (2) to calculate the final phase volume fraction. However, the nonlinear nature of the MUSCL finite volume scheme means that the discrete system does not inherit this 
equivalence. In this work, the no-voids condition is used in place of one of the conservation laws in (1), so that the overall mass/volume of the system is conserved (and to improve the speed of computation). The consequence of this is that the volume fraction of the ignored phase (extracellular material) is no longer guaranteed to have the non-oscillatory properties that the nonlinearities in the numerical approximation are designed to impose. However, this has had no noticeable effect on our model simulations, and $\theta_{i} \in[0,1]$ is maintained without any additional constraints being applied.

\subsection{Phase Velocities and Pressures}

The coupled system of equations given by (20) can be viewed as a multiphase version of the equations used to model Stokes flow. In particular, since they are linear in the unknown variables, $\vec{u}_{i}^{\prime}$ and $p_{4}^{\prime}$, a standard Galerkin finite element approach can be used to approximate the system. Here, a stable, Taylor-Hood, element pair is applied, which uses a continuous, piecewise quadratic representation for the velocities and a continuous, piecewise linear representation for the pressures $[23,37]$. Equations (9) and (10) then lead to

$$
\begin{aligned}
\int_{\Omega} w^{q} \vec{\nabla} \cdot\left(\theta_{i} \boldsymbol{\sigma}_{i}\right) d \vec{x}+\int_{\Omega} w^{q} \vec{F}_{i} d \vec{x} & =0, \quad i=1, \ldots, 4, \\
\int_{\Omega} w^{l} \sum_{i=1}^{N_{p}} \vec{\nabla} \cdot\left(\theta_{i} \vec{u}_{i}\right) d \vec{x} & =0
\end{aligned}
$$

in which $w^{l}$ and $w^{q}$ are, respectively, the standard linear and quadratic Lagrange test functions. After integration by parts, the first of these equations becomes

$$
\oint_{\partial \Omega} w^{q} \theta_{i} \boldsymbol{\sigma}_{i} \cdot \hat{\vec{n}} d s-\int_{\Omega} \vec{\nabla} w^{q} \cdot \theta_{i} \boldsymbol{\sigma}_{i} d \vec{x}+\int_{\Omega} w^{q} \vec{F}_{i} d \vec{x}=0, \quad i=1, \ldots, 4 .
$$

For all degrees of freedom in the interior of the domain the boundary integral is zero because, for the corresponding test function, $w^{q}=0$ on $\Gamma$. For degrees of freedom on the boundary either the normal stress $\boldsymbol{\sigma}_{i} \cdot \hat{\vec{n}}$ is specified, or the equation is replaced by a Dirichlet condition on $\vec{u}_{i}$.

The unknown solution is piecewise polynomial and may be written in terms of trial functions, which are here chosen to be precisely the same as the test functions, so

$$
\vec{u}_{i} \approx \sum_{k=1}^{N_{q}}\left(\vec{u}_{i}\right)_{k} w_{k}^{q}, \quad p \approx \sum_{k=1}^{N_{l}} p_{k} w_{k}^{l}
$$

where $N_{q}$ and $N_{l}$ are the numbers of degrees of freedom associated with the quadratic and linear Lagrange elements, respectively. Each degree of freedom in the discrete system $\left(\left(\vec{u}_{i}\right)_{k}, p_{k}\right)$ has an associated test/trial function with compact support. Integration of the resulting equations leads to a sparse, linear system of equations which is solved here using the MUMPS software package [59]. This is a parallel, sparse, direct solver for large, linear systems of equations (although in this work it is only being used on a single CPU core). 
Remark: Care should be taken in situations where $\theta_{i}=0$ for $i \in\{1,2,3,4\}$ because this will cause at least one of the equations in (20) to become degenerate, creating a singular system. In practice, any equation associated with a degree of freedom for which $\theta_{i}<$ Tol can be treated as though the phase volume fraction is zero, assigning values directly to $\vec{u}_{i}^{\prime}$ instead of trying to include the discretised differential equation in the system to be solved. The effect of this is to introduce into the domain an artificial moving interface within the domain which delineates, for a particular phase $i$, regions in which $\theta_{i}=0$ from regions in which $\theta_{i} \in(0,1)$ and on which a zero normal stress boundary condition is implicitly applied. The chosen value of the tolerance is $T o l=10^{-10}$ and numerical experiments have shown that the simulations are not sensitive to this value once it drops below about $10^{-3}$ (results not presented).

Remark: The use of quadratic Lagrange elements means that information about the midpoints of each edge is required by the code. It is therefore straightforward to apply the finite volume approximation of the mass balance equations (27) on a mesh generated by dividing each triangular cell into four congruent subtriangles. Since solving for the phase velocities and pressures is by far the most time-consuming stage of the simulation, using the fine mesh to provide additional resolution in the representation of the phase volume fractions does not significantly increase run-times: hence this approach is used here.

\subsection{Nutrient Concentration}

The quasi-steady-state, reaction-diffusion equation given by (23) is approximated using a standard Galerkin finite element scheme, with linear elements [23, 37]. This leads to

$$
\int_{\Omega} w^{l_{f}} D_{j} \vec{\nabla}^{2} c_{j} d \vec{x}-\int_{\Omega} w^{l_{f}} q_{j} d \vec{x}=0, \quad j=1, \ldots, N_{d},
$$

which, after integration by parts, gives the weak form

$$
\int_{\partial \Omega} w^{l_{f}} D_{j} \vec{\nabla} c_{j} \cdot \hat{\vec{n}} d s-\int_{\Omega} \vec{\nabla} w^{l_{f}} \cdot D_{j} \vec{\nabla} c_{j} d \vec{x}-\int_{\Omega} w^{l_{f}} q_{j} d \vec{x}=0, \quad j=1, \ldots, N_{d},
$$

where the $w^{l_{f}}$ are now linear test functions defined on the same refined mesh as that used for updating the phase volume fractions. The boundary integral again disappears for internal degrees of freedom because, due to the compact support of the test functions, $w^{l_{f}}=0$ on $\Gamma$.

For degrees of freedom on the boundary, either $\vec{\nabla} c_{j} \cdot \hat{\vec{n}}$ is specified or the equation is replaced by a Dirichlet condition on $c_{j}$. The nutrient is written in terms of the trial functions, i.e.

$$
c \approx \sum_{k=1}^{N_{l_{f}}} c_{k} w_{k}^{l_{f}}
$$

and the integral source term in (32) is approximated using an appropriate quadrature scheme. Since the rest of the scheme is at most second order accurate, no more than second order 
accuracy is required in the quadrature. This leads to a nonlinear system of equations (because $q_{c}$ in Equation (16) is a nonlinear function of $c$ ) which is solved using a matrix-free Newton iteration method [48]. The MUMPS direct solver [59] is again used to solve the linear systems of equations that arises at each iteration.

\subsection{Summary}

The numerical algorithm that we use to solve equations (19), (20) and (23) is applied on an unstructured triangular mesh in which each cell is uniformly divided into four subtriangles to produce a globally refined mesh. The mixed nature of the governing partial differential equations motivates the order in which they are approximated on successive time-steps. The three stages carried out during each time-step are as follows:

1. Given initial values for the phase volume fractions $\theta_{i}$ and the phase velocities $\vec{u}_{i}^{\prime}$, update the cell-averaged values of $\theta_{i}$, nominally stored at the centres of the cells of the globally refined mesh (and interpolated to provide values at the nodes of the refined mesh), using a cell-centred MUSCL finite volume scheme (see Equations (25)-(27)) $[54,77]$. Equation (19) is used to update the cell and blood vessel phase volume fractions in time; the no-voids condition (2) is used to update the volume fraction of the extracellular material $\left(\theta_{4}\right)$.

2. Given the new values of $\theta_{i}$, calculate the values of $\vec{u}_{i}^{\prime}$ at the nodes of the refined mesh (which coincide with the union of the nodes and the edge midpoints of the original mesh) along with the values of $p_{4}^{\prime}$ at the nodes of the original mesh, using a Galerkin finite element scheme with Taylor-Hood elements (see Equations (28)-(30)) [23, 37]. Use linear interpolation to determine the values of $p_{4}^{\prime}$, the pressure of the extracellular material phase, at the nodes of the refined mesh: the remaining phase pressures $p_{i}^{\prime}$ can be found using the algebraic relations given by Equations (20) and (22).

3. Given the new values of $\theta_{i}$, calculate the values of $c^{\prime}$ at the nodes of the refined mesh using a Galerkin finite element scheme with linear elements (31)-(33).

Steps 1-3 are used to evolve the system variables from one time-step to the next and can be repeated to advance the simulation in time. We remark that, since the second and third steps are independent of each other in this model, the order in which the phase velocities and nutrient are updated does not matter.

\section{Numerical Results}

The numerical results presented in this section were obtained by seeding "healthy" tissue in an equilibrium state with a small number of tumour cells. It is assumed that at the equilibrium state: 
- each phase is spatially uniform with zero velocity (i.e. $\left.\vec{u}_{i} \equiv 0, i=1,3,4\right)$;

- there are no tumour cells present (i.e. $\left.\theta_{2} \equiv 0\right)$;

- the volume fraction of healthy cells is such that $\theta_{1}=\theta^{*}=0.6$, the natural cell density, below which the cells do not interact or experience any stress;

- $p_{3}=p_{3}^{*}=0$ and $p_{4}=0$, so $p_{1}=p_{2}=0$ because $\theta_{1}=\theta^{*}$.

Equations (19), (2), (20) and (23) then reduce to the system of nonlinear equations given by

$$
\begin{aligned}
\theta^{*} \theta_{4}\left(\frac{c^{\prime}}{c_{p}^{*}+c^{\prime}}\right)-k_{2,1}^{*} \theta^{*}\left(\frac{c_{c_{1}}^{*}+c^{\prime}}{c_{c_{2}}^{*}+c^{\prime}}\right) & =0, \\
-k_{3}^{*} \theta_{3} \mathcal{H}\left(-p_{c r i t}^{*}, \epsilon_{3}^{*}\right)+k_{4}^{*} \theta^{*} \theta_{3}\left(\frac{\theta_{4}}{\varepsilon+\theta_{4}}\right)\left(\frac{c^{\prime}}{\left(c_{a}^{*}+c^{\prime}\right)^{2}}\right) & =0, \\
\theta^{*}+\theta_{3}+\theta_{4} & =1, \\
\theta_{3}\left(1-c^{\prime}\right)-k_{6,1}^{*} \theta^{*} c^{\prime}-k_{7,1}^{*} \theta^{*} \theta_{4}\left(\frac{c^{\prime}}{c_{p}^{*}+c^{\prime}}\right) & =0 .
\end{aligned}
$$

These equations are then solved for the unknown model variables $\theta_{3}, \theta_{4}$ and $c^{\prime}$, and one model parameter, chosen arbitrarily here to be the angiogenesis rate constant $k_{4}^{*}$. The chosen solution must also satisfy $\theta_{3}, \theta_{4}, c^{\prime} \in[0,1]$ and $k_{4}^{*} \geq 0$ to be valid physically. The solution for the remaining parameter values shown in Table 1 is given by

$$
\begin{gathered}
\theta_{1}=0.6, \quad \theta_{2}=0.0, \quad \theta_{3}=0.01749783, \quad \theta_{4}=0.3825022 \\
c^{\prime}=0.2532031, \quad p_{1}=p_{2}=p_{3}=p_{4}=0.0
\end{gathered}
$$

with $k_{4}^{*}=0.002944900$ (all to 7 significant figures).

\subsection{Single Tumour Seeded in a Circular Domain}

For the first set of numerical simulations a circular domain of radius 16 was used and covered by an unstructured, triangular mesh containing 2349 nodes and 4539 elements. At $t=0$ a small tumour was seeded at the centre of the healthy tissue so that

$$
\theta_{2}(x, y, t=0)= \begin{cases}0.05 \cos ^{2}(\pi r / 2) & \text { for } r \leq 1 \\ 0 & \text { otherwise }\end{cases}
$$

where $r=\sqrt{x^{2}+y^{2}}$. To compensate for the addition of tumour cells, the initial distribution of healthy cells was modified to be

$$
\theta_{1}(x, y, 0)=0.6-\theta_{2}(x, y, 0)
$$

while all other variables were initialised throughout the domain using the values given in (35). The boundary conditions were chosen as follows. 


\begin{tabular}{|c|c|l|}
\hline Parameter & Value & Description \\
\hline$k_{1,2}^{*}$ & $2.0 \dagger$ & Tumour cell birth rate \\
$k_{2,1}^{*}$ & 0.15 & Healthy cell death rate \\
$k_{2,2}^{*}$ & $0.075 \dagger$ & Tumour cell death rate \\
$k_{3}^{*}$ & 0.1 & Vessel occlusion rate \\
$k_{4}^{*}$ & 0.0029449 & Angiogenesis rate \\
$k_{6,1}^{*}$ & 0.01 & Nutrient consumption rate (healthy cell baseline) \\
$k_{6,2}^{*}$ & 0.01 & Nutrient consumption rate (tumour cell baseline) \\
$k_{7,1}^{*}$ & 0.1 & Nutrient consumption rate (healthy cell birth) \\
$k_{7,2}^{*}$ & $k_{7,1}^{*} \times k_{1,2}^{*}$ & Nutrient consumption rate (tumour cell birth) \\
$c_{p}^{*}$ & 0.25 & Cell birth rate dependence on nutrient \\
$c_{c_{1}}^{*}, c_{c_{2}}^{*}$ & $0.2,0.1$ & Cell death rate dependence on nutrient \\
$c_{a}^{*}$ & 0.05 & Angiogenesis rate dependence on nutrient \\
$p_{c r i t}^{*}$ & 0.3 & Critical pressure for vessel occlusion \\
$\epsilon_{3}^{*}$ & 0.2 & Smoothness of occlusion pressure dependence \\
$\varepsilon$ & 0.01 & Angiogenesis rate dependence on ECM \\
$\Lambda^{*}$ & $0.1 \dagger$ & Cell tension constant \\
$\mu_{i}^{*}$ & $10.0 \dagger$ & Phase dynamic shear viscosities $\left(i=1, \ldots, N_{p}\right)$ \\
$\lambda_{i}^{*}$ & $-\frac{2}{3} \mu_{i}^{*}$ & Phase bulk viscosities $\left(i=1, \ldots, N_{p}\right)$ \\
$d_{i j}^{*}$ & 1.0 & Interphase drag coefficients $\left(i, j=1, \ldots, N_{p}, j \neq i\right)$ \\
$D_{c}^{*}$ & $1.0 \dagger$ & Nutrient diffusion coefficient \\
\hline
\end{tabular}

Table 1: Dimensionless parameter values for the benchmark simulations: $\dagger$ indicates values which vary in later experiments. 
- For Equations (20) impose

$$
\boldsymbol{\sigma}_{i}^{\prime} \cdot \hat{\vec{n}}=0, \quad i=1,2,3, \quad \text { and } \quad \vec{u}_{4}^{\prime}=0
$$

on the whole of $\Gamma$. This allows flow of cell and blood vessel phases through the boundary.

- For Equation (23) impose

$$
\vec{\nabla}^{\prime} c^{\prime} \cdot \hat{\vec{n}}=0
$$

on the whole of $\Gamma$.

- For Equations (19) impose

$$
\theta_{i}=\theta_{i}^{\infty}, \quad i=1,2,3,4
$$

on $\Gamma_{i}^{\text {Inflow }}$, the region of $\Gamma$ for which $\vec{u}_{i}^{\prime} \cdot \hat{\vec{n}}<0$ ( $\hat{\vec{n}}$ being the outward-pointing unit normal to the boundary).

These conditions are inevitably artificial, in the sense that interactions with tissue outside the computational domain are neglected. However, numerical experiments have shown that the choice of boundary condition (e.g. Dirichlet, imposing the equilibrium values from (35), or Neumann, imposing zero stress or flux) has little effect on the tumour's growth until the proliferating rim gets close to the domain boundary. Any cases presented below for which this is not the case are highlighted.

The values of the remaining parameters are given in Table 1, where we assume that the tumour cells proliferate and die at, respectively, double and half the rate of their normal counterparts.

The evolution of the volume fraction of tumour cells under these conditions is shown in Figure 5. The initial cluster expands rapidly to produce a high density of tumour cells at the centre of the domain, where the phase volume fraction exceeds the natural cell density, i.e. $\theta_{2}>\theta^{*}$. The tumour then starts to spread outwards and eventually the tumour cells in the centre start to die, creating a proliferating rim of tumour cells, behind which a necrotic core develops. This qualitative behaviour is representative of that seen in the majority of simulations carried out with this model. The mechanisms which generate this characteristic growth pattern can be inferred from Figures 6 and 7, which show how the corresponding phase fluxes and pressures evolve, and Figure 8, which shows how the nutrient concentration develops.

Since the tumour cells are assumed to proliferate more rapidly and die less readily than healthy cells, at early times tumour cells undergo net proliferation, absorbing extracellular material as they do so. This causes $\theta_{4}$ to fall locally, reducing the net birth rate of the healthy cells and leading to their depletion. In addition, the tumour pushes the healthy cells in front of it as it grows, some of the volume being replaced by extracellular material which 

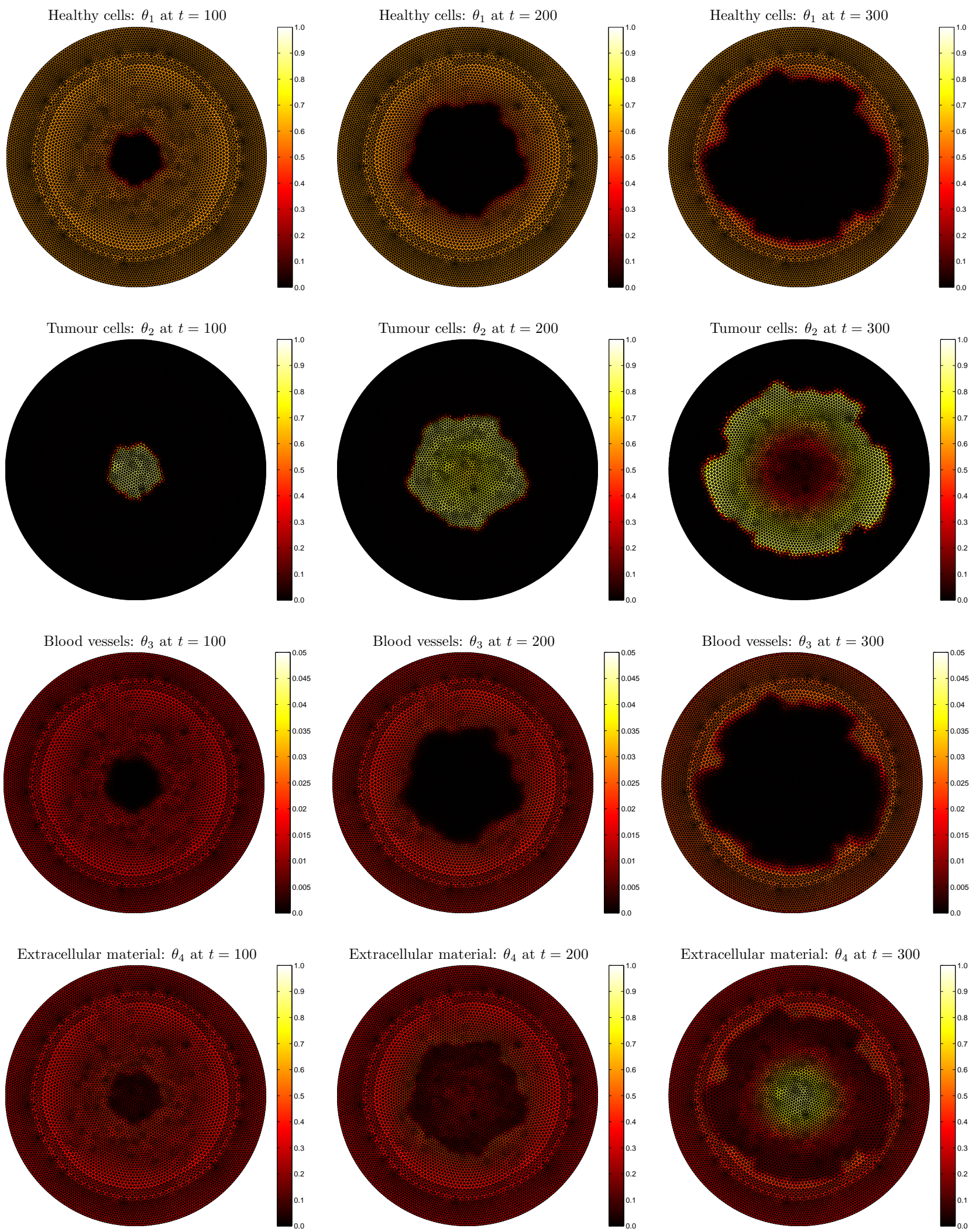

Figure 5: Snapshots of the evolution of the phase volume fractions: healthy cells, $\theta_{1}$ (top row); tumour cells, $\theta_{2}$ (second row); blood vessels, $\theta_{3}$ (third row); extracellular material, $\theta_{4}$ (bottom row). Time increases from left to right: parameter values are as in Table 1. 
Healthy cells: $\theta_{1} \vec{u}_{1}^{\prime}$ at $t=100$

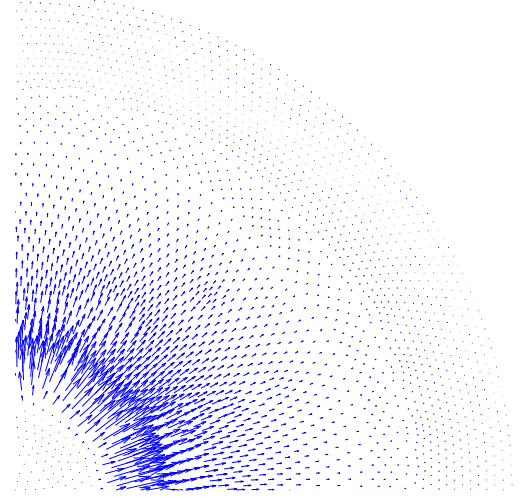

Tumour cells: $\theta_{2} \vec{u}_{2}^{\prime}$ at $t=100$

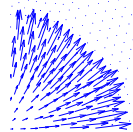

Extracellular material: $\theta_{4} \vec{u}_{4}^{\prime}$ at $t=100$

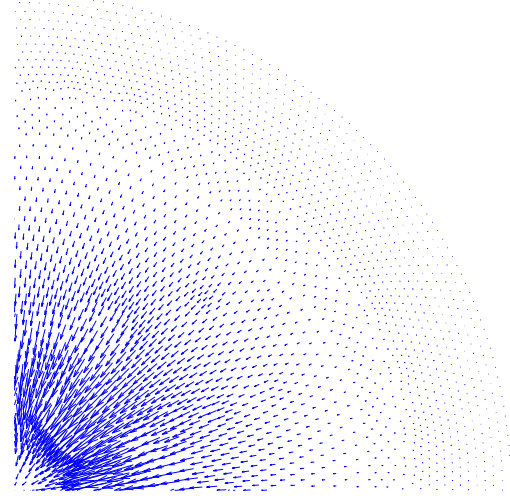

Healthy cells: $\theta_{1} \vec{u}_{1}^{\prime}$ at $t=200$

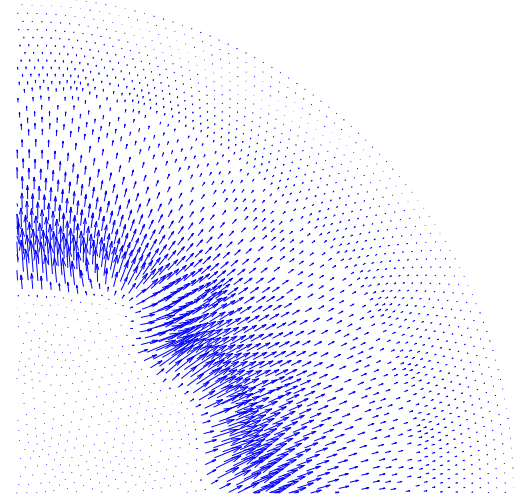

Tumour cells: $\theta_{2} \vec{u}_{2}^{\prime}$ at $t=200$

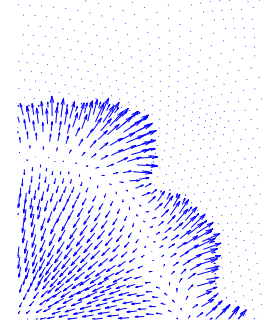

Extracellular material: $\theta_{4} \vec{u}_{4}^{\prime}$ at $t=200$

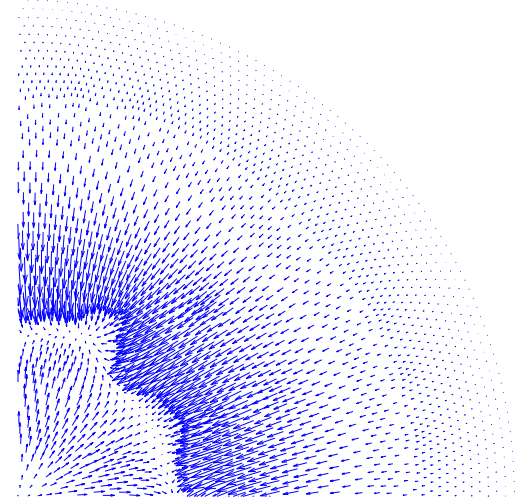

Healthy cells: $\theta_{1} \vec{u}_{1}^{\prime}$ at $t=300$

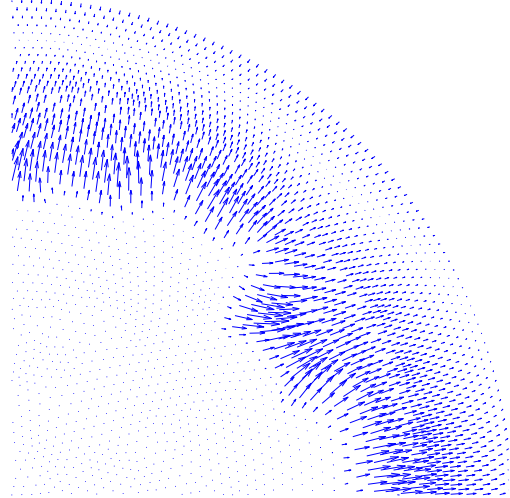

Tumour cells: $\theta_{2} \vec{u}_{2}^{\prime}$ at $t=300$

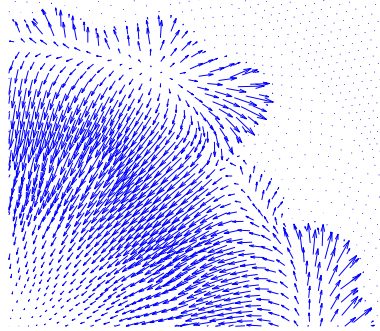

Extracellular material: $\theta_{4} \vec{u}_{4}^{\prime}$ at $t=300$

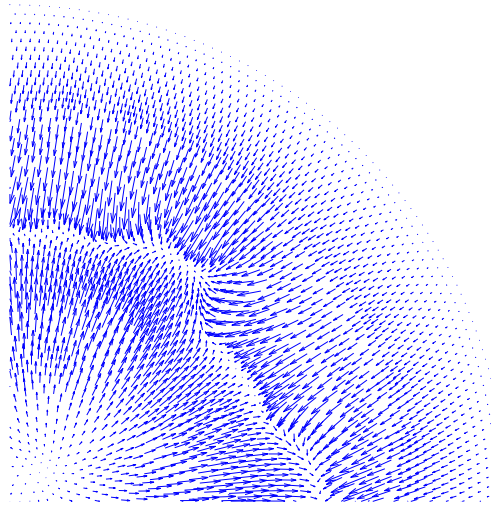

Figure 6: Snapshots of the evolution of the phase fluxes: healthy cells, $\theta_{1} \vec{u}_{1}^{\prime}$ (top row); tumour cells, $\theta_{2} \vec{u}_{2}^{\prime}$ (second row); blood vessels, $\theta_{3} \vec{u}_{3}^{\prime}$ (third row); extracellular material, $\theta_{4} \vec{u}_{4}^{\prime}$ (bottom row). Time increases from left to right: parameter values are as in Table 1 . The arrows are scaled by the magnitude of the flux vector and all are plotted to the same scale: the blood vessel phase is not shown because the arrows are not visible at this scale. 

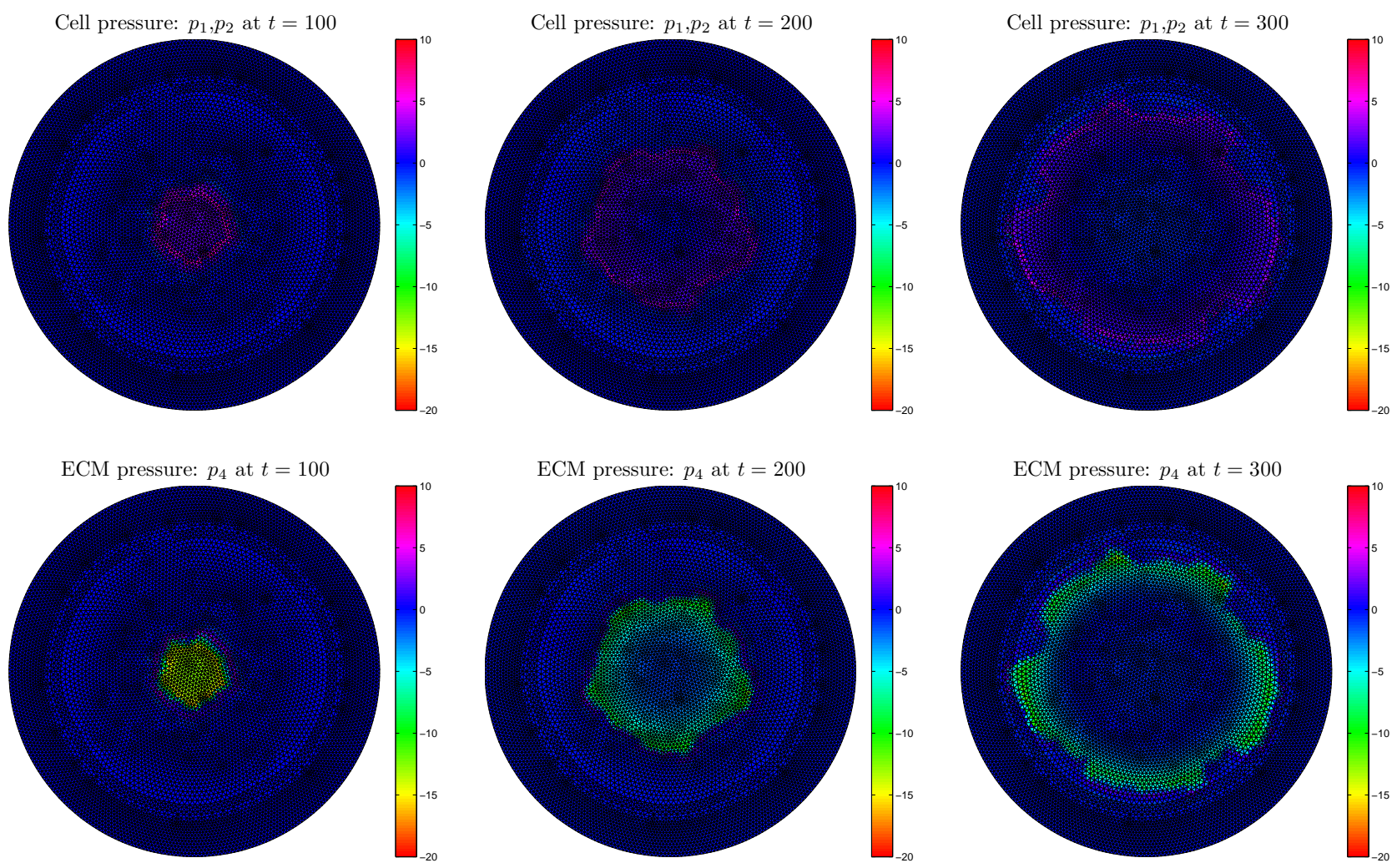

Figure 7: Snapshots of the evolution of the phase pressures: healthy/tumour cells, $p_{1}^{\prime}=$ $p_{2}^{\prime}$ (top row); extracellular material, $p_{4}^{\prime}$ (bottom row). Time increases from left to right: parameter values are as in Table 1 . The pressures for the blood vessel phase automatically vanish and are, therefore, not included.
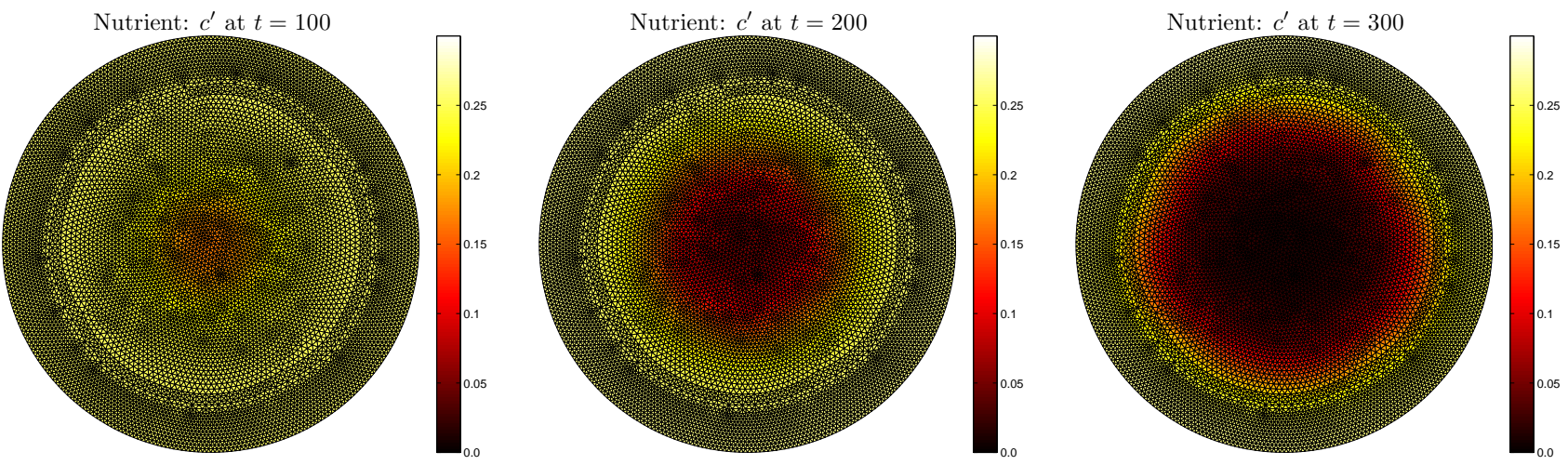

Figure 8: Snapshots of the evolution of the nutrient concentration, $c^{\prime}$. Time increases from left to right: parameter are values as in Table 1. 
is simultaneously drawn towards the tumour ( $c f$. Figure 6). At the same time, the high pressures generated by the high density of tumour cells causes vessel occlusion, restricting the nutrient supply to the interior of the tumour. This further exacerbates the imbalance between birth and death for the healthy cells whose volume fraction is rapidly driven to zero (a state from which they never recover).

As the tumour grows, the size of the region in which the blood vessels are occluded also expands, the angiogenic response being too weak to perfuse the entire tissue, causing the centre of the tumour to become progressively starved of nutrient. Tumour cells in the interior then start to die and a necrotic core, consisting mainly of extracellular material, develops behind a rim of proliferating cells. The healthy cells continue to be pushed by the advancing tumour rim and replaced by extracellular material, which fuels the growth of the tumour cells. The proliferating rim also draws extracellular material towards it from inside the tumour, replacing it with tumour cells which move towards the core where they die due to lack of nutrient (as illustrated in Figure 6).

The moving front of proliferating tumour cells is characteristically very sharp, and typically preceded by a small rise in ECM phase volume fraction, with a far smoother transition behind the front to the necrotic core. The front is not completely circular because the computational mesh and the discretised initial conditions are not radially symmetric. The extent of deviation from radial symmetry may be interpreted in terms of the sensitivity of the system to inhomogeneities in the tissue.

\subsection{Parameter Sensitivity Analysis}

In order to assess how the rate of tumour growth depends on the values of the model parameters, the size of the tumour is measured by estimating the distance of the tumour front from the centre of the computational domain. This is done by calculating $R_{\max }(t)$, the furthest distance of the contour $\theta_{2}=\theta^{*}=0.6$ from the origin, and plotting graphs of $R_{\max }(t)$ against time (see Figures 9, 10 and 11). In all plots, growth rates corresponding to the parameter values stated in Table 1 are drawn with a solid black line with small dots. Those parameters marked with daggers in Table 1 are varied, as indicated in the figure legends. Note that in Figure 11, all of the viscosities are assumed to be identical in the top left graph, and both cell viscosities are assumed identical in the top right graph.

At the start of each numerical experiment, the time taken for the tumour cell density to exceed the natural cell density appears to be independent of the material properties of the tissue. This is indicated in Figures 9 and 11 by the coincidence of the growth curves at early times (until the radius of the tumour reaches approximately one, indicated by the first symbol on each graph). However, the rate of this initial stage of growth does depend on the birth and death rates of the tumour cells (see the early stages of the growth curves shown in Figure 10): faster birth and/or slower death of the tumour cells causes the initial small, 

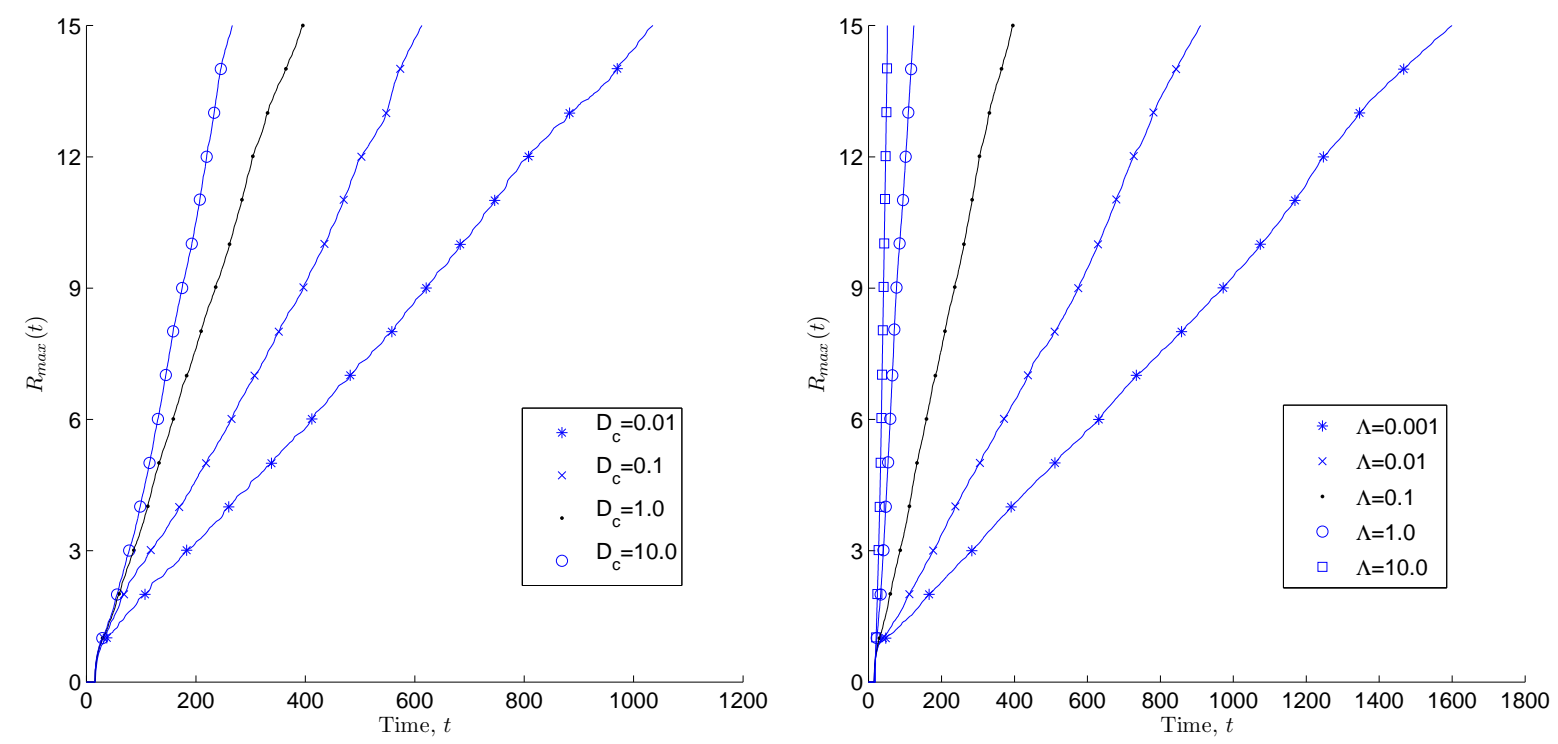

Figure 9: Series of plots showing how the tumour growth rate depends on the nutrient diffusion coefficient $D_{c}$ (left) and the cell tension constant, $\Lambda$ (right). Each curve shows the evolution of $R_{\max }(t)$ over time.
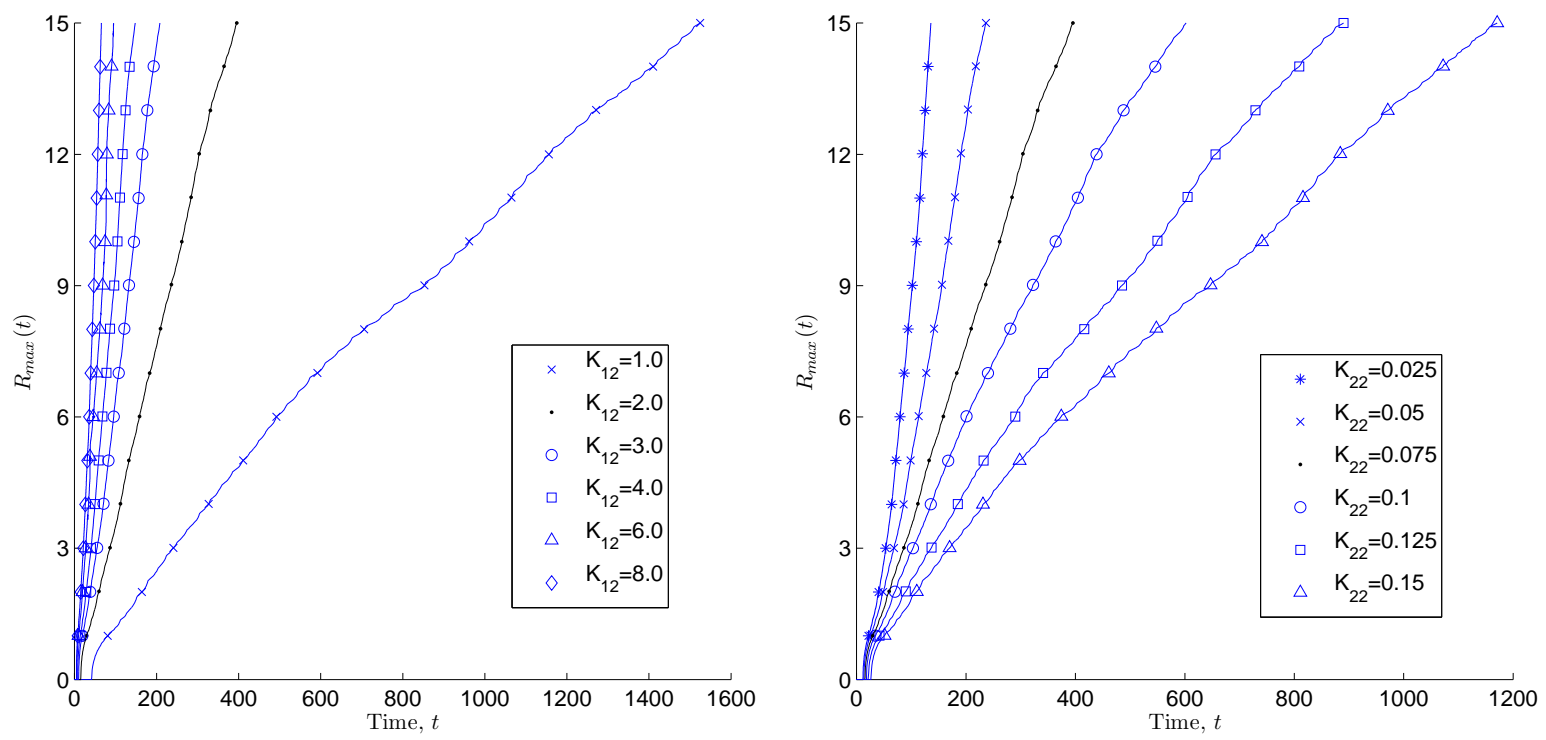

Figure 10: Series of plots showing how the tumour growth rate depends on the tumour cell birth rate, $k_{12}$ (left) and the tumour cell death rate, $k_{22}$ (right). Each curve shows the evolution of $R_{\max }(t)$ over time. 

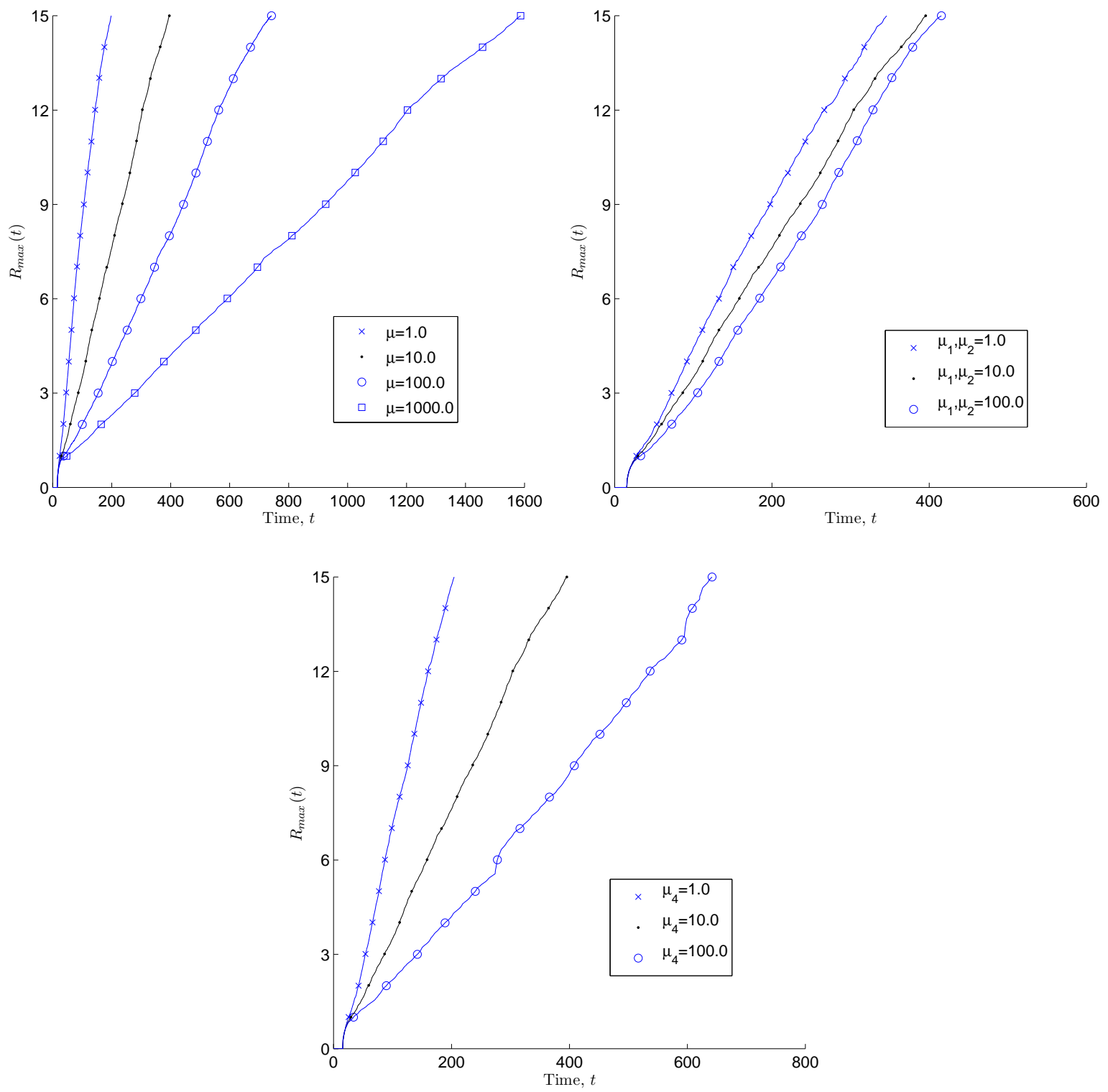

Figure 11: Series of plots showing how the tumour growth rate depends on the phase viscosities, $\mu_{i}^{*}$ : varying all of the phase viscosities (top left); varying only the viscosity of the normal and cancer cells (top right); varying only the ECM phase viscosity (bottom). Each curve shows the evolution of $R_{\max }(t)$ over time. 
but dense, cluster of tumour cells to form more rapidly.

In all cases the tumour cell volume fraction in the centre of the domain rapidly reaches a maximum value (typically in the range $[0.8,0.9]$ ) which is maintained in the proliferating region as the tumour grows. After this initial transient, the speed with which the sharp front of tumour cells travels remains approximately constant, resembling a steady travelling wave and suggesting that the tumour growth rate predicted by this model is typically linear.

While the qualitative features seen in Figure 5 persist when the model parameters are varied, the rate at which the tumour grows and the width of the proliferating rim can change significantly. The sensitivity of these quantities to the model parameters is discussed in more detail below.

The moving front remains sharply defined in all of the situations tested here. This suggests that the model distinguishes clearly between the growing tumour and the exterior tissue, a view reinforced by the fact that the volume fraction of healthy cells drops rapidly to zero behind the moving front and never recovers. Hence, we conclude that within this multiphase modelling framework, it is often reasonable to use the approach proposed in [14, 64] and to model the tumour separately from its surroundings, with a moving boundary representing the interface between the tumour and the healthy tissue and appropriate boundary conditions accounting for the influence of the environment. However, we anticipate that this would not always be the case: for example, when simulating the elimination of a vascular tumour in response to a blood-borne drug that targets proliferative cells. In such situations, the sharp front would most likely be smoothed out in the manner of the transition from the proliferating rim to the necrotic core behind the moving front in the simulations shown in Section 4.1.

Varying the nutrient diffusion rate, $D_{c}^{*}$ : Both the growth rate of the tumour ( $c f$. Figure 9) and the width of the proliferating rim increase as $D_{c}^{*}$ is increased. This is because higher diffusion coefficients increase the size of the domain of influence of the blood-borne nutrients. As Figure 12 shows, this increases the net proliferation rate of the tumour cells, and hence the width of the viable rim. For large values of $D_{c}^{*}\left(e . g . D_{c}^{*}=10.0\right)$ the tumour cells remain densely packed behind the rim, with no necrotic core having developed by the end of the simulations. Further increases in $D_{c}^{*}$ cause the length scales over which diffusion acts to become so large that boundary effects influence the solution (result not included). Conversely, for small values of $D_{c}^{*}\left(e . g . D_{c}^{*}=0.1,0.01\right)$, for which the proliferating rim is much narrower and the invading front becomes more convoluted (trails of blood vessels remain within the tumour which can later be reinforced by angiogenesis), the pressures close to the moving front are lower and, as a result, the front moves more slowly.

Varying the cell tension parameter, $\Lambda^{*}$ : Larger values for $\Lambda^{*}$ corresponds to cells that respond more readily to dense packing (when $\theta>\theta^{*}$ ). The pressures in these tumours are lower, and their cells correspondingly less densely packed, and they grow more rapidly 

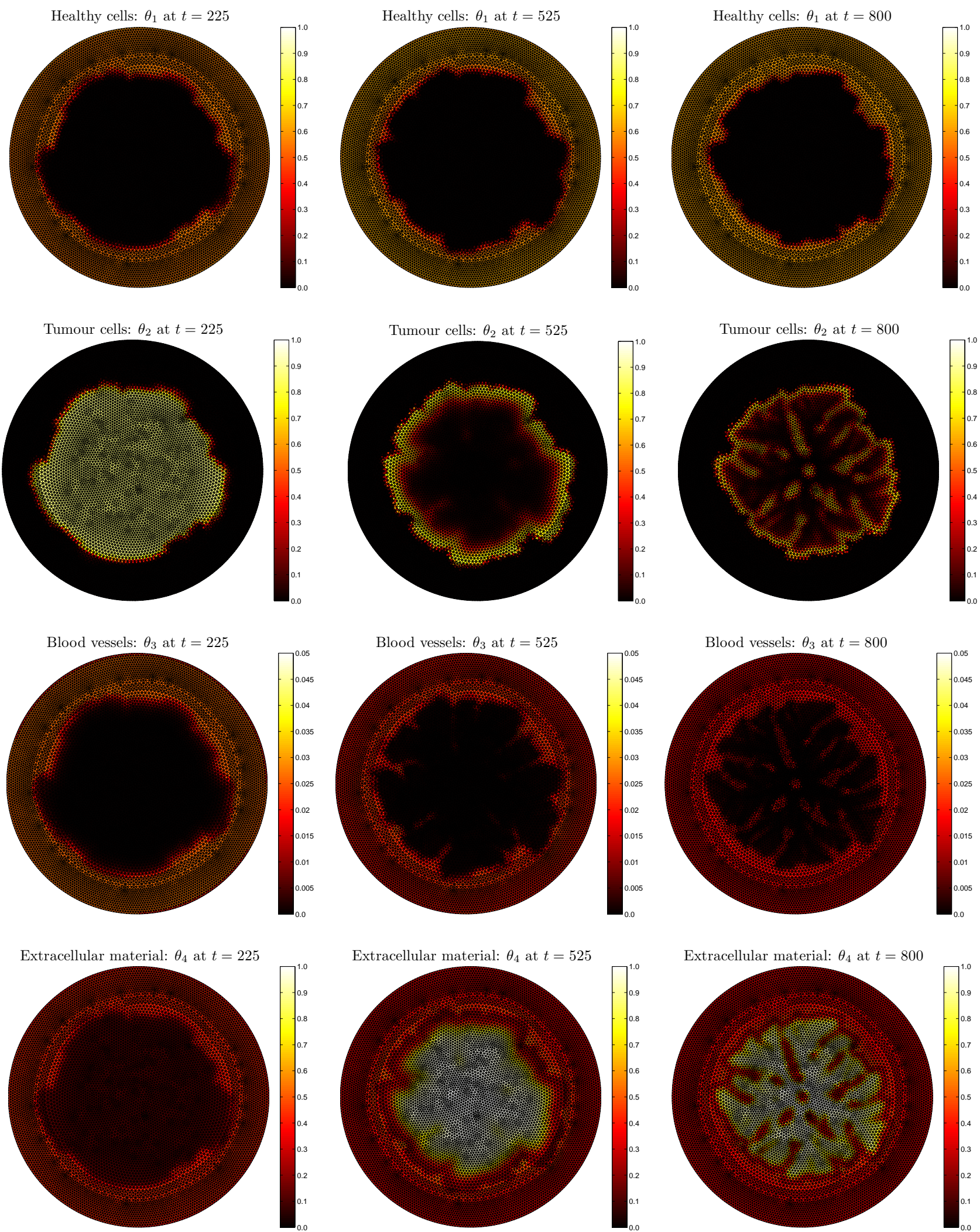

Figure 12: Snapshots of the evolution of the phase volume fractions: healthy cells, $\theta_{1}$ (top row); tumour cells, $\theta_{2}$ (second row); blood vessels, $\theta_{3}$ (third row); extracellular material, $\theta_{4}$ (bottom row). Parameter values are as in Table 1, except for the nutrient diffusion coefficient, which decreases from left to right: $D_{c}^{*}=10.0$ (left), $D_{c}=0.1$ (middle), $D_{c}=0.01$ (right). For each value of $D_{c}^{*}$, results are presented at a time for which $R_{\max }(t) \approx 12$. 
Varying the tumour cell birth and death rates, $k_{12}^{*}$ and $k_{22}^{*}$ : Figure 10 shows that the tumour's growth rate increases as $k_{12}^{*}$ increases and/or $k_{22}^{*}$ decreases.

Varying the phase viscosities, $\mu_{i}^{*}$ : In general, increasing the viscosity of any or all of the phases causes the cell pressure to increase while the tumour's growth rate slows and its structure remains unchanged ( $c f$. Figure 11). Additionally, as Figure 13 illustrates, the proliferating rim becomes slightly wider and more circular. The growth rate is most sensitive to variation in the viscosity of the extracellular material phase and almost completely insensitive to the viscosity of the blood vessel phase. This remains the case in situations where the blood vessels take up a much higher proportion of the tissue (results not shown).
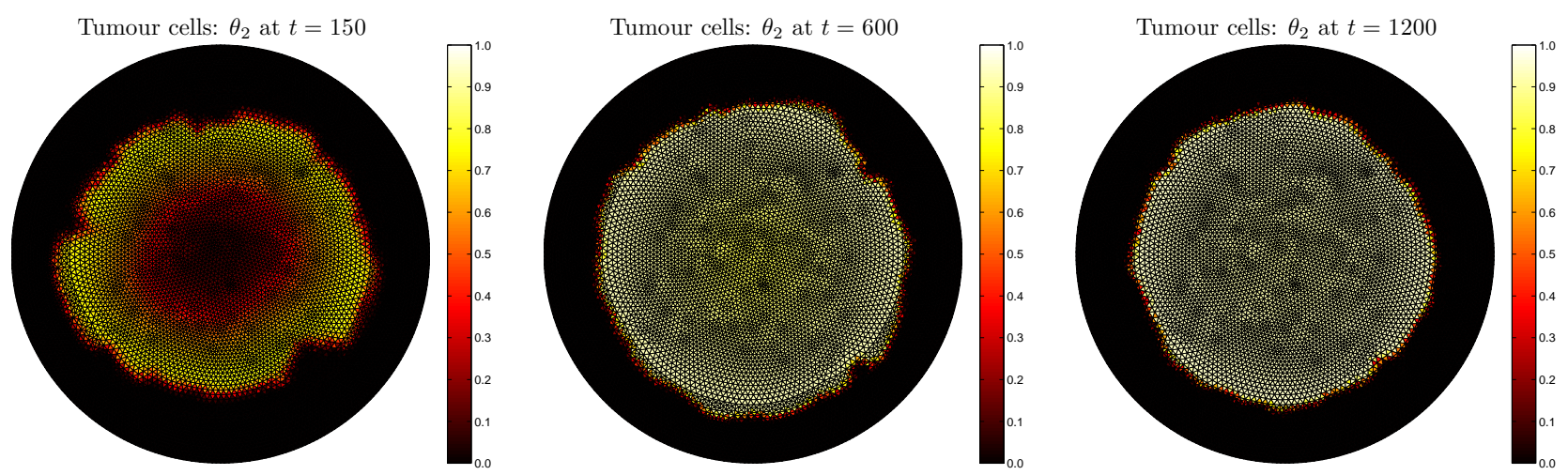

Figure 13: Snapshots of the evolution of the tumour cell phase volume fraction, $\theta_{2}$. Parameter values are as in Table 1, except for the phase viscosities, which increase from left to right: $\mu_{i}^{*}=1.0$ (left), $\mu_{i}^{*}=100.0$ (middle), $\mu_{i}^{*}=1000.0$ (right), $i=1,2,3,4$. For each value of $\mu_{i}^{*}$, results are presented at a time for which $R_{\max }(t) \approx 12$.

\subsection{Interacting Tumours}

Since our computational model tracks the moving front of tumour cells on a fixed computational mesh rather than by following a free boundary delineating the tumour from its surrounding tissue, it is straightforward to seed the virtual tissue with multiple tumours. To illustrate this versatility, we now use our computational model to simulate the evolution of a vascular tissue which is initially seeded with two small, circular tumours centred at $(x, y)=(2.5,2.5)$ and $(x, y)=(-2.5,0)$. Accordingly we prescribe

$$
\theta_{2}(x, y, t=0)= \begin{cases}0.05 \cos ^{2}\left(\pi r_{1} / 2\right) & \text { for } r_{1} \leq 1 \\ 0.05 \cos ^{2}\left(\pi r_{2} / 2\right) & \text { for } r_{2} \leq 1 \\ 0 & \text { otherwise }\end{cases}
$$

where $r_{1}=\sqrt{(x-2.5)^{2}+(y-2.5)^{2}}$ and $r_{2}=\sqrt{(x+2.5)^{2}+y^{2}}$, and setting $\theta_{1}=0.6-\theta_{2}$. 
The evolution of the tumour cell volume fraction is depicted in Figure 14 where the two tumours can be seen to merge, and then to grow as one, eventually producing a tumour whose structure is similar to that generated when the tissue is initially seeded with a single cluster of tumour cells (compare Figures 14 and 5).
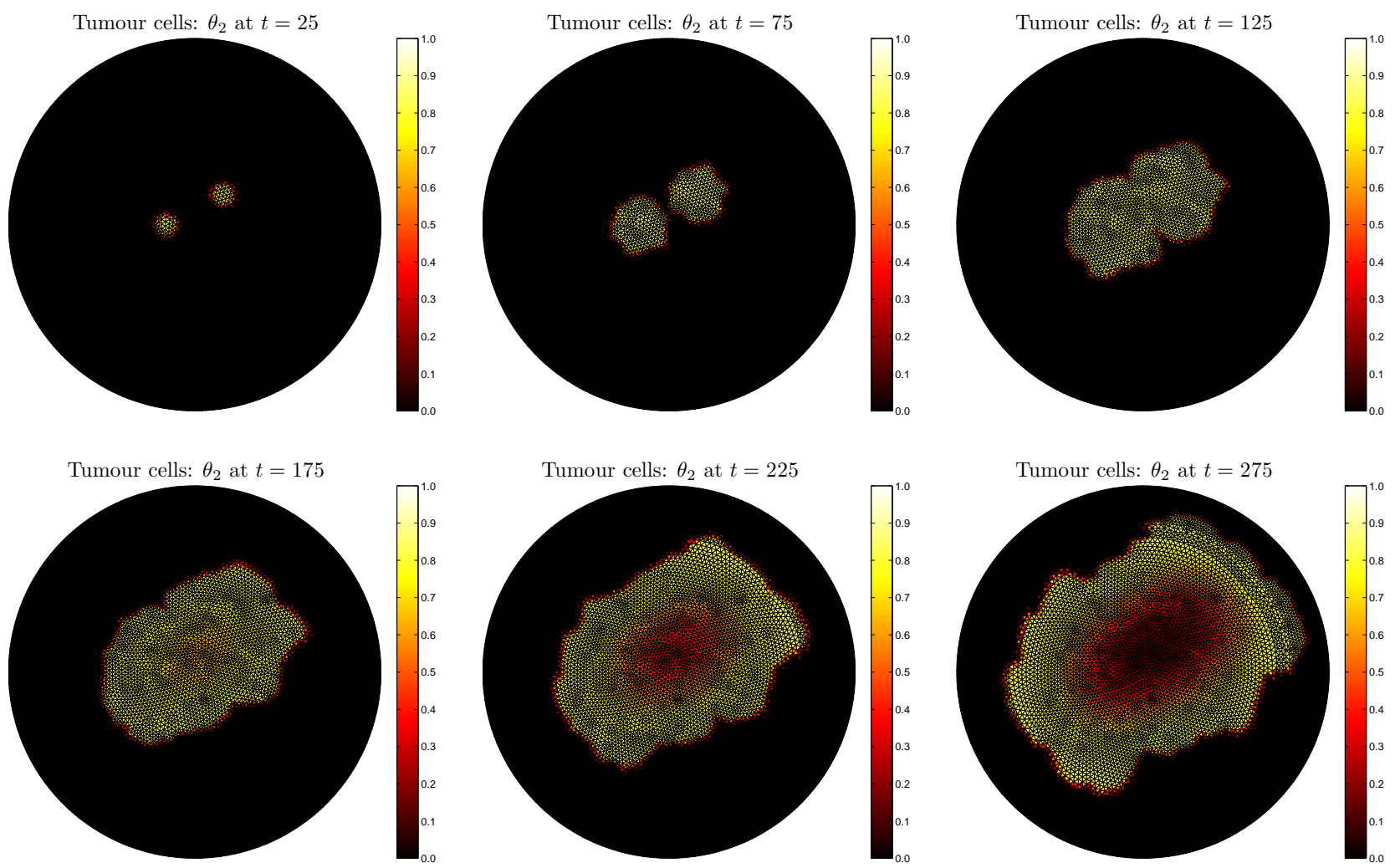

Figure 14: Snapshots of the evolution of the tumour cell phase volume fraction, $\theta_{2}$. Time increases from left to right and then top to bottom: parameter values are as in Table 1 . Two tumours are seeded in the healthy tissue.

\subsection{Tapered Domain}

The final set of numerical simulations are carried out on a tapered domain, formed by cutting a wedge out of the bottom right hand corner of the rectangle $[0,16] \times[0,4]$, as illustrated in Figure 15. The top and bottom boundaries are treated as solid walls, so that at the boundary nodes $\vec{u}_{i}^{\prime}=0$ is imposed in (20) for all phases, $\vec{\nabla}^{\prime} c^{\prime} \cdot \hat{\vec{n}}=0$ is substituted in (23). The boundary conditions imposed at the left and right ends of the domain are the same as those used in the previous test cases, except that it is no longer necessary to impose $\vec{u}_{4}^{\prime}=0$ because of the solid walls elsewhere.

The domain is covered by an unstructured triangular mesh, with 2496 nodes and 4740 cells, and the initially healthy tissue ( $c f$. the values in (35)) is specified by setting $\theta_{1}(x, y, 0)=$ 
$0.6-\theta_{2}(x, y, 0)$ where

$$
\theta_{2}(x, y, t=0)= \begin{cases}0.05 \cos ^{2}(\pi r / 2) & \text { for } r \leq 1 \\ 0 & \text { otherwise }\end{cases}
$$

and $r=\sqrt{(x-8)^{2}+(y-2)^{2}}$. As before, we use the parameter values given in Table 1 .

The simulation results presented in Figure 16 exhibit behaviour similar to that described earlier. There is an initial period of rapid growth, after which a necrotic core forms behind the proliferating rim of tumour cells. The solid walls prevent the tumour from spreading upwards and downwards, but it continues to spread laterally as time progresses.

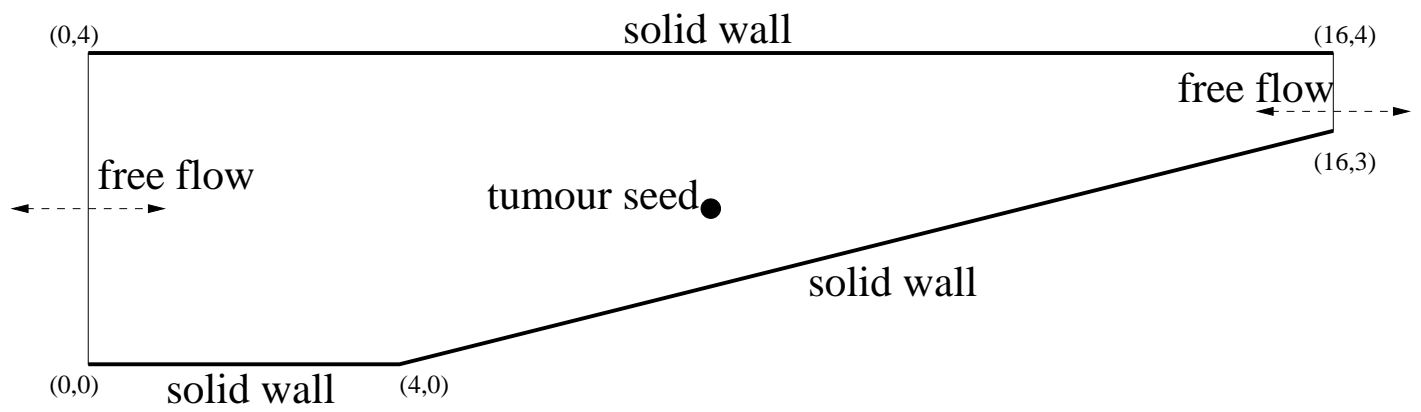

Figure 15: Geometry and boundary conditions for the tapered domain.

\section{Discussion}

This paper describes a multidimensional, multiphase model of vascular tumour growth, and a computational approach for its solution which uses no artificial stabilisation techniques and is capable of handling irregular geometries. It extends earlier work by Breward, Byrne and Lewis $[13,14]$ and has the following features.

- The model includes four phases: two cell types, blood vessels and extracellular material. In particular, because it distinguishes between tumour cells and healthy cells, interactions between the two species can be incorporated. Each phase is assumed to behave like a viscous fluid. A diffusible nutrient is included (as in [13] but not [14]), so that the blood vessels can have a non-local influence on the surrounding tissue.

- The tumour's evolution is simulated within a fixed computational domain and its boundary is captured on the mesh as a diffuse interface instead of tracking it and using it as the boundary of the domain, which would be computationally challenging for the more irregular fronts seen in Section 4. It also allows the tumour to interact with the normal/healthy cells constituting the surrounding tissue.

- Using a mixed finite volume/finite element approach on unstructured triangular meshes makes it straightforward to implement nonuniform meshes and solve the governing 
Tumour cells: $\theta_{2}$ at $t=50$

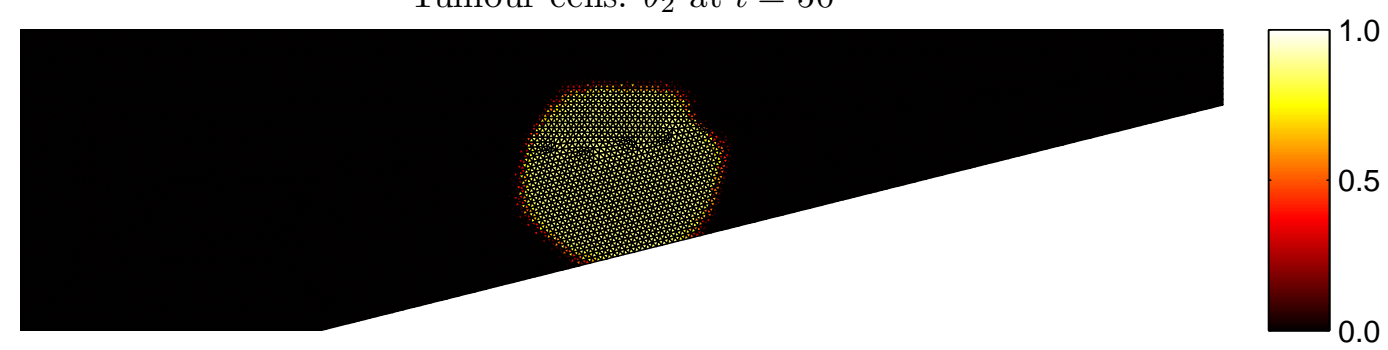

Tumour cells: $\theta_{2}$ at $t=100$

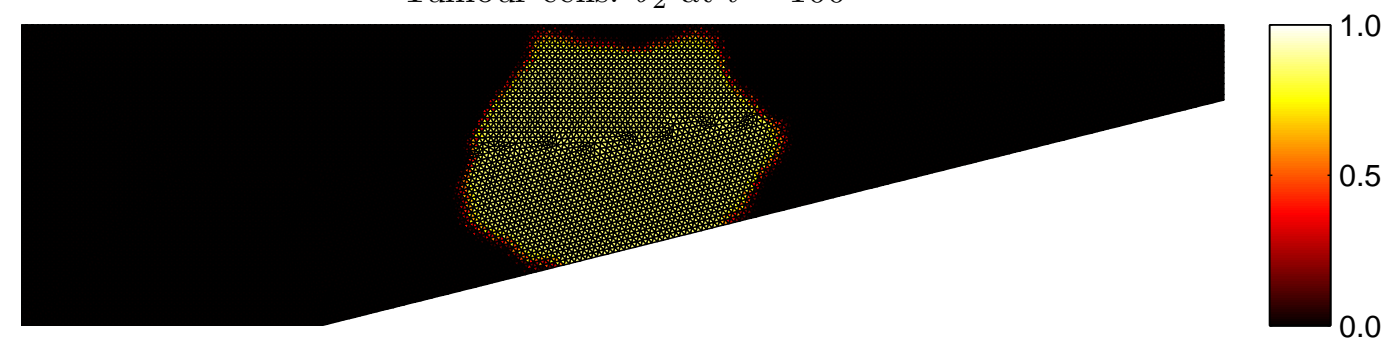

Tumour cells: $\theta_{2}$ at $t=200$

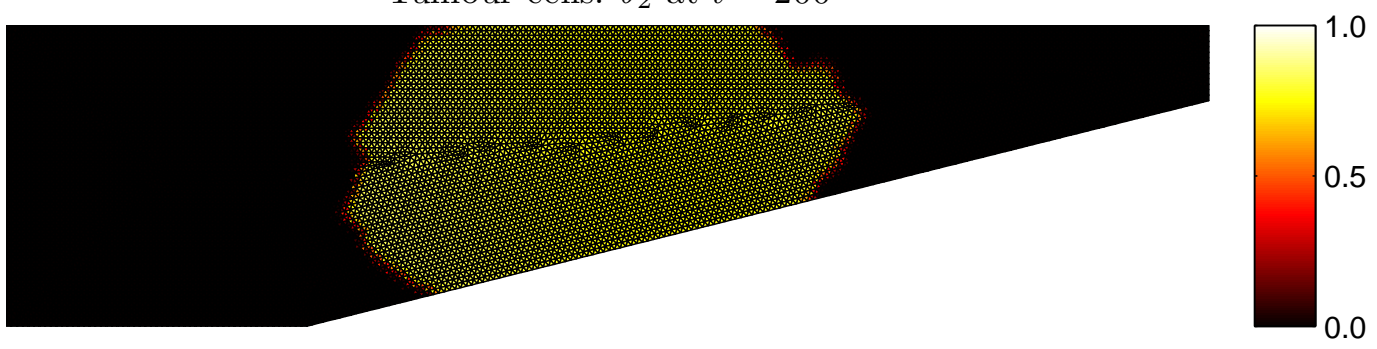

Tumour cells: $\theta_{2}$ at $t=400$

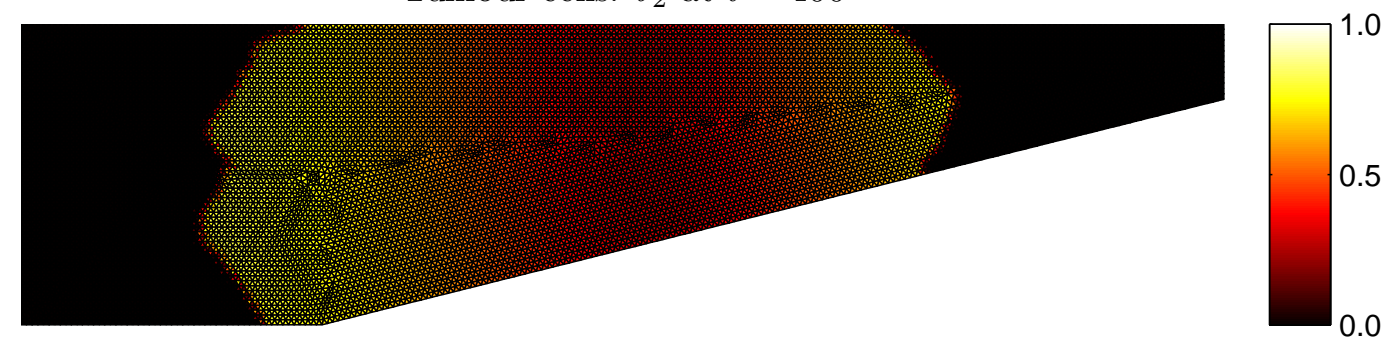

Figure 16: Snapshots of the evolution of the tumour cell phase volume fraction, $\theta_{2}$, when a single, circular tumour is seeded in a tapered domain. Time increases from top to bottom: parameter values are as in Table 1. 
equations on complex multidimensional geometries. A Newton solver allows for nonlinear terms to be included in the model.

Both the mathematical model and the numerical approximation generalise straightforwardly to tetrahedral meshes in three space dimensions. However this would require a parallel implementation (and appropriate computing resources) to obtain results with high enough resolution to be informative. Three-dimensional simulations of a simpler multiphase model have been carried out by Lowengrub, Cristini and coauthors $[28,81,82]$. They were able to resolve the tumour boundary by applying sophisticated computational techniques (adaptive multigrid) to a finite difference scheme on block-structured meshes, for a model in which all of the phase velocities are assumed to be the same.

In Section 4 the model is used to simulate the growth of a tumour initiated by seeding a small cluster of tumour cells in "healthy" tissue in an equilibrium state. In each case, giving the tumour cells a higher birth rate and/or a lower death rate led to the rapid appearance of a small region with a high density of tumour cells which then spread in to the surrounding tissue. The increased pressures generated by the growing tumour mass caused occlusion of the blood vessels, inhibiting the supply of nutrient to the interior of the tumour and leading to the development of a necrotic core behind an outwardly moving, proliferating rim. This is a characteristic pattern commonly seen in tumour growth.

The model consistently predicted that the tumour radius increases approximately linearly with time, though the speed with which the front moves depends strongly on the model parameters. Increasing $D_{c}^{*}$, the nutrient diffusion coefficient, $\Lambda^{*}$, the sensitivity of the cells to dense packing, or $k_{12}$, the tumour cell birth rate, or decreasing $k_{22}$, the tumour cell death rate or any of the phase viscosities, all increased the rate of tumour growth.

The mathematical model presented describes physical phenomena using very simple functional forms, which provide a qualitative representation of the expected behaviour, and a limited number of phases. It is not difficult to propose potential improvements, e.g. elastic or viscoelastic models for the cells, extensions to investigate response to treatment, more realistic models of cell metabolism, decomposition of the blood vessels into two phases (for mature vessels and angiogenic sprouts), and the inclusion of an additional diffusible species or angiogenic factor which stimulates endothelial cell proliferation and elicits a chemotactic response from the angiogenic sprouts. Additional diffusible species that distinguish between metabolites such as oxygen, glucose and lactic acid could be introduced, thereby permitting an investigation of the impact on the simulated growth dynamics of using more realistic models of cell metabolism, including the switch from aerobic to anaerobic respiration and the associated production of lactic acid [24, 31, 32]. Alternatively, additional phases could be included to represent the extracellular matrix in which the normal and cancerous cells reside and the lymph network into which excess extracellular fluid drains (and through which tumour fragments can migrate, or metastasise, to other parts of the body) [50]. The lymph network evolves in a similar manner to the vascular network, new lymph vessels being stimulated by the production of appropriate growth factors and existing vessels collapsing under 
excess pressure [10]. Of particular interest is investigating how the interplay between the evolving vascular and lymph networks affects the tumour's development [47]. However, before any such model can be used to generate quantitative predictions, it will have to be validated and parameterised against experimental data.

Advances in imaging technology mean that magnetic resonance images are now routinely used to obtain coarse-grained information about the spatial extent of solid tumours. Such data, combined with functional images that provide spatially-resolved information on rates of, for example, oxygen and glucose delivery and consumption are now being integrated with simple continuum models of solid tumour growth to estimate parameter values that characterise the tumours. This approach is also being used to predict patient responses to specific treatment protocols $[74,75,76]$. In future work, we aim to validate and parameterise multiphase models of the type presented in this paper against such biomedical imaging data.

The computational algorithms used are all standard in other application areas, and known to have excellent properties when applied to the individual components of the system. However, this is the first time they have been combined to simulate this type of coupled, multiphase model of fluid flow. An upwind "Total Variation Diminishing" (TVD) finite volume scheme is chosen to approximate the hyperbolic mass balance equations to ensure that (i) the phase volume fractions remain in the interval $[0,1]$ without the need for any artificial smoothing and (ii) mass is conserved. The momentum balance equations lead to a generalised Stokes flow which is approximated using Taylor-Hood finite elements, an inherently stable algorithm for finding the phase velocities and pressures. Finite elements are also used with a Newton iteration to approximate the nonlinear equations governing nutrient diffusion.

These well-understood, robust, algorithms have been chosen to give confidence that our qualitative interpretation of the simulations is not corrupted by spurious, numericallycreated, artefacts. While the mathematical models remain in the early stages of development it is unlikely that more sophisticated techniques will be required to improve the order of accuracy of the approximation. However, improvements in speed and efficiency would be beneficial.

For example, the use of a direct solver on a single processor machine to find the discrete phase velocities and pressures has restricted the resolution of the mesh and the size of the computational domain used in the simulations. Direct solvers for large systems are expensive in terms of both cpu time and memory so, to apply this model on larger domains, with a wider range of parameter values (to adequately resolve all of the features, such as the proliferating rim) or in three space dimensions, an iterative approach would be needed. This would also require investigation of appropriate preconditioners for the system given by the momentum balance equations. When combined with other techniques for improving the speed with which simulations can be carried out to a specified accuracy, e.g. adaptive meshing, multigrid algorithms, parallel implementations, this should lead to a tool which will help us to fully understand the behaviour of these multiphase models, and hence improve their design. Additional stabilisation techniques may also be introduced, e.g. the DEVSS 
approach proposed by Guénette and Fortin [39] for viscoelastic flows, to further widen the range of parameter values for which reliable numerical results can be obtained.

In summary, a mathematical model has been presented which describes vascular tumour growth and allows interaction between the tumour, the vasculature and the surrounding tissue. It is based on existing multiphase models but the computational model developed here demonstrates that the framework can be implemented in multiple space dimensions. There is also considerable scope adapting our model to describe other biomedical applications in, for example, tissue engineering and wound healing.

\section{Acknowledgements}

This publication was based on work supported in part by Award No. KUK-013-04, made by King Abdullah University of Science and Technology (KAUST).

\section{References}

[1] T. Alarcón, H.M. Byrne, P.K. Maini, A cellular automaton model for tumour growth in inhomogeneous environment, J Theor Biol, 225:257-274, 2003.

[2] T. Alarcón, H.M. Byrne, P.K. Maini, Towards whole-organ modelling of tumour growth, Prog Biophys Mol Bio, 85:451-472, 2004.

[3] T. Alarcón, H.M. Byrne, P.K. Maini, A multiple scale model for tumor growth, Multiscale Model Sim, 3(2):440-475, 2005.

[4] D. Ambrosi, L. Preziosi, Cell adhesion mechanisms and stress relaxation in the mechanics of tumours, Biomech Model Mechan, 8:397-413, 2009.

[5] R.P. Araujo, D.L.S. McElwain, A history of the study of solid tumour growth: The contribution of mathematical modelling, B Math Biol, 66:1039-1091, 2004.

[6] R.P. Araujo, D.L.S. McElwain, A mixture theory for the genesis of residual stresses in growing tissues: I. A general formulation, SIAM J Appl Math, 65:1261-1284, 2005.

[7] R.P. Araujo, D.L.S. McElwain, A mixture theory for the genesis of residual stresses in growing tissues: II. Solutions to the biphasic equations for a multicell spheroid, SIAM J Appl Math, 66:447-467, 2005.

[8] T.J. Barth, D.C. Jesperson, The design and application of upwind schemes on unstructured meshes, AIAA Paper 89-0366, 1989. 
[9] E.L. Bearer, J.S. Lowengrub, Y.-L. Chuang, H.B. Frieboes, F. Jin, S.M. Wise, M. Ferrari, D.B. Agus, V. Cristini, Multiparameter computational modeling of tumor invasion, Cancer Res, 69:4493-4501, 2009.

[10] N.J.P. Beasley, R. Prevo, S. Banerji, R.D. Leek, J. Moore, P. van Trappen, G. Cox, A.L. Harris, D.G. Jackson, Intratumoral lymphangiogenesis and lymph node metastasis in head and neck cancer, Cancer Res, 62:1315-1320, 2002.

[11] F. Billy, B. Ribba, O. Saut, H. Morre-Trouilhet, T. Colin, D. Bresch, J.-P. Boissel, E. Grenier, J.-P. Flandrois, A pharmacologically based multiscale mathematical model of angiogenesis and its use in investigating the efficacy of a new cancer treatment strategy, J Theor Biol, 260:545-562, 2009.

[12] C.J.W. Breward, H.M. Byrne, C.E. Lewis, Modelling the interactions between tumour cells and a blood vessel in a microenvironment within a vascular tumour, Eur J Appl Math, 12:529-556, 2001.

[13] C.J.W. Breward, H.M. Byrne, C.E. Lewis, The role of cell-cell interactions in a twophase model for avascular tumour growth, J Math Biol, 45:125-152, 2002.

[14] C.J.W. Breward, H.M. Byrne, C.E. Lewis, A multiphase model describing vascular tumour growth, B Math Biol, 65:609-640, 2003.

[15] H.M. Byrne, J.R. King, D.L.S. McElwain, L. Preziosi, A two-phase model of solid tumour growth, Appl Math Lett, 16:567-573, 2003.

[16] H.M. Byrne, M.R. Owen, A new interpretation of the Keller-Segel model based on multiphase modelling, J Math Biol, 49:604-626, 2004.

[17] H.M. Byrne, L. Preziosi, Modelling solid tumour growth using the theory of mixtures, Math Med Biol, 20:341-366, 2003.

[18] M.A.J. Chaplain, L. Graziano, L. Preziosi, Mathematical modelling of the loss of tissue compression responsiveness and its role in solid tumor development, Math Med Biol, 23:197-229, 2006.

[19] M.A.J. Chaplain, S.R. McDougall, A.R.A. Anderson, Mathematical modeling of tumorinduced angiogenesis, Annu Rev Biomed Eng, 8:233-257, 2006.

[20] V. Cristini, X. Li, J.S. Lowengrub, S.M. Wise, Nonlinear simulations of solid tumor growth using a mixture model: Invasion and branching, J Math Biol, 58(4-5):723-763, 2009.

[21] F. Demirag, E. Unsal, A. Yilmaz, A. Caglar, Prognostic significance of vascular endothelial growth factor, tumour necrosis and mitotic activity index in malignant pleural mesothelioma, Chest, 128:3382-3387, 2005. 
[22] S. Dormann, A. Deutsch, Modeling of self-organized avascular tumor growth with a hybrid cellular automaton, In Silico Biol, 2:393-406, 2002.

[23] H.C. Elman, D.J. Silvester, A.J. Wathen, Finite Elements and Fast Iterative Solvers: With Applications in Incompressible Fluid Dynamics, OUP Oxford, 2005.

[24] J.S. Fang, R.D. Gillies, R.A. Gatenby, Adaptation to hypoxia and acidosis in carcinogenesis and tumour progression, Semin Cancer Biol, 18(5):330-337, 2008.

[25] J. Folkman, Role of angiogenesis in tumour growth and metastases, Semin Oncol, 29(Suppl 16):15-19, 2002.

[26] J. Folkman, R. Cotran, Relation of vascular proliferation to tumour growth, Intl Rev Exp Pathol, 16:207-248, 1976.

[27] J. Folkman, M. Hochberg, Self-regulation of growth in three dimensions, J Exp Med, 138(4):745-753, 1973.

[28] H.B. Frieboes, F. Jin, Y.-L. Chuang, S.M. Wise, J.S. Lowengrub, V. Cristini, Threedimensional multispecies nonlinear tumor growth - II: Tumor invasion and angiogenesis, J Theor Biol, 264(4):1254-1278, 2010.

[29] H.B. Frieboes, J.S. Lowengrub, S.M. Wise, X. Zheng, P. Macklin, E.L. Bearer, V. Cristini, Computer simulation of glioma growth and morphology, NeuroImage, 37:S59S70, 2007.

[30] J. Galle, L. Preziosi, A. Tosin, Contact inhibition of growth described by a multiphase and an individual cell-based model, Appl Math Lett, 22:1483-1490, 2009.

[31] R.A. Gatenby, R.J. Gillies, Why do cancers have high aerobic glycolysis? Nat Rev Cancer, 4:891-899, 2004.

[32] R.A. Gatenby, K. Smallbone, P.K. Maini, F. Rose, J. Averill, R.B. Nagle, L. Worrall, R.J. Gillies, Cellular adaptations to hypoxia and acidosis during somatic evolution of breast cancer, Br J Cancer, 97(5):646-653, 2007.

[33] J.L. Gevertz, S. Torquato, Modeling the effects of vasculature evolution on early brain tumor growth, J Theor Biol, 243:517-531, 2006.

[34] J. Gevertz, S. Torquato, Growing heterogeneous tumors in silico, Phys Rev E, 80:051910, 2009.

[35] H.P. Greenspan, Models for the growth of a solid tumour by diffusion, Stud Appl Math, 1:317-340, 1972 .

[36] H.P. Greenspan, On the growth and stability of cell cultures and solid tumours. J Theor Biol, 56:229-242, 1976. 
[37] P.M. Gresho, R.L. Sani, Incompressible Flow and the Finite Element Method, WileyBlackwell, 2000.

[38] J.E.F. Green, S.L. Waters, K.M. Shakesheff, H.M. Byrne, A mathematical model of liver cell aggregation in vitro, B Math Biol, 71:906-930, 2009.

[39] R. Guénette, M. Fortin, A new mixed finite element method for computing viscoelastic flows, J Non-Newton Fluid, 60:27-52, 1995.

[40] X. He, M. Dembo, On the mechanics of the first cleavage division of the sea urchin egg, Exp Cell Res, 233:252-273, 1997.

[41] M. Herant, M. Dembo, Form and function in cell motility: From fibroblasts to keratocytes, Biophys J, 98:1408-1417, 2010.

[42] M. Herant, V. Heinrich, M. Dembo, Mechanics of neutrophil phagocytosis: Experiments and quantitative models, J Cell Sci, 119:1903-1913, 2006.

[43] M. Herant, A. Marganski, M. Dembo, The mechanics of neutrophils: Synthetic modeling of three experiments, Biophys J, 84:3389-3413, 2003.

[44] C.S. Hogea, B.T. Murray, J.A. Sethian, Simulating complex tumor dynamics from avascular to vascular growth using a general level-set method, J Math Biol, 53:86-134, 2006.

[45] T.L. Jackson, H.M. Byrne, A mathematical model to study the effects of drug resistance and vasculature on the response of solid tumors to chemotherapy, Math Biosci, 164:1738,2000 .

[46] T.L. Jackson, H.M. Byrne, A mechanical model of tumor encapsulation and transcapular spread, Math Biosci, 180:307-328, 2002.

[47] R.K. Jain, R.T. Tong, L.L. Munn, Effect of vascular normalization by antiangiogenic therapy on interstitial hypertension, peritumor edema, and lymphatic metastasis: insights from a mathematical model, Cancer Res, 67:2729-2735, 2007.

[48] C.T. Kelley, Iterative Methods for Linear and Nonlinear Equations, SIAM, Philadelphia, 1995.

[49] L.A. Kunz-Schughart, Multicellular tumour spheroids: intermediates between monolayer culture and in vivo tumour, Cell Biol Int, 23(3):157-161, 1999.

[50] D. Leclers, K. Durand, A. Dutour, G. Barrière, J. Monteil, M. Rigaud, F. Sturtz, Lymphatic vessels and cancer, Med Sci (Paris), 21(10):839-847, 2005.

[51] W.P. Leenders, B. Kusters, R.M. de Waal, Vessel co-option: how tumours obtain blood supply in the absence of sprouting angiogenesis, Endothelium, 9(2):83-87, 2002. 
[52] G. Lemon, J.R. King, Multiphase modelling of cell behaviour on artificial scaffolds: Effects of nutrient depletion and spatially nonuniform porosity, Math Med Biol, 24:57$83,2007$.

[53] G. Lemon, J.R. King, H.M. Byrne, O.E. Jensen, K.M. Shakesheff, Mathematical modelling of engineered tissue growth using a multiphase porous flow mixture theory, $J$ Math Biol, 52:571-594, 2006.

[54] R.J. LeVeque, Finite Volume Methods for Hyperbolic Problems, Cambridge University Press, 2002.

[55] J.S. Lowengrub, H.B. Frieboes, F. Jin, Y.-L. Chuang, X. Li, P. Macklin, S.M. Wise, V. Cristini, Nonlinear modelling of cancer: Bridging the gap between cells and tumours, Nonlinearity, 23:R1-R91, 2010.

[56] S.R. Lubkin, T.L. Jackson, Multiphase mechanics of capsule formation in tumors, $J$ Biomech Eng-T ASME, 124:237-243, 2002.

[57] P. Macklin, S. McDougall, A.R.A. Anderson, M.A.J. Chaplain, V. Cristini, J. Lowengrub, Multiscale modelling and nonlinear simulation of vascular tumour growth, $J$ Math Biol, 58:765-798, 2009.

[58] J. Moreira, A. Deutsch, Cellular automation models of tumor development: a critical review, Adv Complex Syst, 5(2-3):247-267, 2002.

[59] MUMPS: a MUltifrontal Massively Parallel sparse direct Solver, http://graal.ens-lyon.fr/MUMPS/ [last accessed 6th February 2012].

[60] R.D. O'Dea, S.L. Waters, H.M. Byrne, A two-fluid model for tissue growth within a dynamic flow environment, Eur J Appl Math, 19:607-634, 2008.

[61] R.D. O’Dea, S.L. Waters, H.M. Byrne, A multiphase model for tissue construct growth in a perfusion bioreactor, Math Med Biol, 27(2):95-127, 2010.

[62] M.E. Orme, M.A.J. Chaplain, A mathematical model of vascular tumour growth and invasion, Math Comput Model, 23(10):43-60, 1996.

[63] J.M. Osborne, R.D. O’Dea, J.P. Whiteley, H.M. Byrne, S.L. Waters, The influence of bioreactor geometry and the mechanical environment on engineered tissues, $J$ Biomech Eng-T ASME, 132(5):051006, 2010.

[64] J.M. Osborne, J.P. Whiteley, A numerical method for the multiphase viscous flow equations, Comput Method Appl M, 199:3402-3417, 2010.

[65] M.R. Owen, T. Alarćon, P.K. Maini, H.M. Byrne, Angiogenesis and vascular remodelling in normal and cancerous tissues, J Math Biol, 58(4-5):689-721, 2009. 
[66] M.R. Owen, I.J. Stamper, M. Muthana, G.W. Richardson, J. Dobson, C.E. Lewis, H.M. Byrne, Mathematical modeling predicts synergistic antitumor effects of combining a macrophage-based, hypoxia-targeted gene therapy with chemotherapy, Cancer Res, 71(8):2826-2837, 2011.

[67] A.A. Patel, E.T. Gawlinski, S.K. Lemieux, R.A. Gatenby, A cellular automaton model of early time tumor growth and invasion: The effects of native tissue vascularity and increased anaerobic tumor metabolism, J Theor Biol, 213:315-331, 2001.

[68] S.M. Peirce, Computational and mathematical modeling of angiogenesis, Microcirculation, 15:739-751, 2008.

[69] H. Perfahl, H.M. Byrne, T. Chen, V. Estrella, T. Alarcón, A. Lapin, R.A. Gatenby, R.J. Gillies, M.C. Lloyd, P.K. Maini, M. Reuss, M.R. Owen, Multiscale modelling of vascular tumour growth in 3D: The roles of domain size and boundary conditions, PLoS ONE, 6(4):e14790, 2011.

[70] L. Preziosi (Ed.), Cancer Modelling and Simulation, Chapman Hall/CRC Press, 2003.

[71] L. Preziosi, A.R. Tosin, Multiphase modeling of tumor growth and extracellular matrix interaction: Mathematical tools and applications, J Math Biol, 58:625-656, 2009.

[72] T. Roose, S.J. Chapman, P.K. Maini, Mathematical models of avascular tumor growth, SIAM Rev, 49:179-208, 2007.

[73] T. Roose, P.A. Netti, L.L. Munn, Y. Boucher, R. Jain, Solid stress generated by spheroid growth using a linear poroelasticity model, Microvasc Res, 66:204-212, 2003.

[74] K.R. Swanson, G. Chakraborty, C.H. Wang, R. Rockne, H.L. Harpold, M. Muzi, T.C. Adamsen, K.A. Krohn, A.M. Spence, Complementary but distinct roles for MRI and 18F-fluoromisonidazole PET in the assessment of human glioblastomas, J Nucl Med, 50(1):36-44, 2009.

[75] K.R. Swanson, R.C. Rockne, J. Claridge, M.A. Chaplain, E.C. Alvord Jr, A.R. Anderson, Quantifying the role of angiogenesis in malignant progression of gliomas: in silico modeling integrates imaging and histology, Cancer Res, 71(24):7366-7375, 2011.

[76] M.D. Szeto, G. Chakraborty, J. Hadley, R. Rockne, M. Muzi, E.C. Alvord Jr, K.A. Krohn, A.M. Spence, K.R. Swanson, Quantitative metrics of net proliferation and invasion link biological aggressiveness assessed by MRI with hypoxia assessed by FMISOPET in newly diagnosed glioblastomas, Cancer Res, 69(10):4502-4509, 2009.

[77] J.C. Tannehill, D.A. Anderson, R.H. Pletcher, Computational Fluid Mechanics and Heat Transfer, Taylor \& Francis, 1997. 
[78] A. Tosin, L. Preziosi, Multiphase modeling of tumor growth with matrix remodeling and fibrosis, Math Comput Model, 52(7-8):979-976, 2010.

[79] P. Tracqui, Biophysical models of tumor growth, Rep Prog Phys, 72:056701, 2009.

[80] B. van Leer, Towards the ultimate conservative difference scheme V. A second order sequel to Godunov's method, J Comput Phys, 32:101-136, 1979.

[81] S.M. Wise, J.S. Lowengrub, V. Cristini, An adaptive multigrid algorithm for simulating solid tumour growth using mixture models, Math Comput Model, 53:1-20, 2011.

[82] S.M. Wise, J.S. Lowengrub, H.B. Frieboes, V. Cristini, Three-dimensional multispecies nonlinear tumor growth - I: Model and numerical method, J Theor Biol, 253:524-543, 2008 .

[83] X. Zheng, S.M. Wise, V. Cristini, Nonlinear simulation of tumor necrosis, neovascularization and tissue invasion via an adaptive finite-element/level-set method, $B$ Math Biol, 67:211-259, 2005. 



\section{RECENT REPORTS}

$71 / 11$ Nonuniqueness in a minimal model for cell motility

Gallimore

Whiteley

Waters

King

Oliver

72/11 Symmetry of uniaxial global Landau-de Gennes minimizers in the

Henao theory of nematic liquid crystals

Majumdar

73/11 Filling of a Poisson trap by a population of random intermittent

Bressloff searchers

Newby

01/12 Mechanical growth and morphogenesis of seashells

Moulton

Goriely

Chirat

02/12 How linear features alter predator movement and the functional response

McKenzie

Merrill

Spiteri

Lewis

03/12 The Fourier transform of tubular densities

Prior

Goriely

04/12 Numerical studies of homogenization under a fast cellular flow.

Iyer

Zygalakis

05/12 Solute transport within porous biofilms: diffusion or dispersion?

Davit

Byrne

Osborne

Pitt-Francis

Gavaghan

Quintard

65/11 Adaptive Finite Element Method Assisted by Stochastic Simulation of Chemical Systems

Cotter

Vejchodsky

Erban

06/12 Effects of intrinsic stochasticity on delayed reaction-diffusion patterning systems

Woolley

Baker

Gaffney

Maini

Seirin-Lee

07/12 Axial Dispersion via Shear-enhanced Diffusion in Colloidal Sus-

Griffiths pensions

Stone

08/12 Qualitative Analysis of an Integro-Differential Equation Model of

Jaina Periodic Chemotherapy

Byrne

09/12 Modeling Stem/Progenitor Cell-Induced Neovascularization and Oxygenation

Jain

Moldovan

Byrne

10/12 Allee Effects May Slow the Spread of Parasites in a Coastal Marine

Krkošek Ecosystem

Connors

Lewis

Poulin 
12/12 Modelling temperature-dependent larval development and subse-

Wheeler quent demographic Allee effects in adult populations of the alpine butterfly Parnassius smintheus

Bampfylde

Lewis

13/12 Putting "space" back into spatial ecology

Fortin

Peres-Neto

Lewis

14/12 Wildlife disease elimination and density dependence

Potapova

Merrill

Lewis

15/12 Spreading Speed, Traveling Waves, and Minimal Domain Size in Impulsive Reaction-diffusion Models

Lewis

$\mathrm{Li}$

16/12 MCMC methods for functions modifying old algorithms to make them faster

Cotter

Roberts

Stuart

White

17/12 Weyl Geometry and the Nonlinear Mechanics of Distributed Point

Yavari Defects

Goriely

18/12 A note on oblique water entry

Moore

Howison

Ockendon

Oliver

19/12 Calculus on surfaces with general closest point functions

März

Macdonald

20/12 Multiple equilibria in a simple elastocapillary system

Taroni

Vella

Copies of these, and any other OCCAM reports can be obtained from:

Oxford Centre for Collaborative Applied Mathematics Mathematical Institute

24 - 29 St Giles'

Oxford

OX1 3LB

England

www.maths.ox.ac.uk/occam 